This item was submitted to Loughborough's Research Repository by the author.

Items in Figshare are protected by copyright, with all rights reserved, unless otherwise indicated.

\title{
Hydrodynamic permeability of aggregates of porous particles with an
} impermeable core

\section{PLEASE CITE THE PUBLISHED VERSION}

http://dx.doi.org/10.1016/j.cis.2010.08.004

PUBLISHER

(C) Elsevier

VERSION

AM (Accepted Manuscript)

LICENCE

CC BY-NC-ND 4.0

\section{REPOSITORY RECORD}

Deo, Satya, Anatoly Filippov, Ashish Tiwari, Sergey Vasin, and Victor Starov. 2011. "Hydrodynamic Permeability of Aggregates of Porous Particles with an Impermeable Core". figshare.

https://hdl.handle.net/2134/8443. 
This item was submitted to Loughborough's Institutional Repository (https://dspace.lboro.ac.uk/) by the author and is made available under the following Creative Commons Licence conditions.

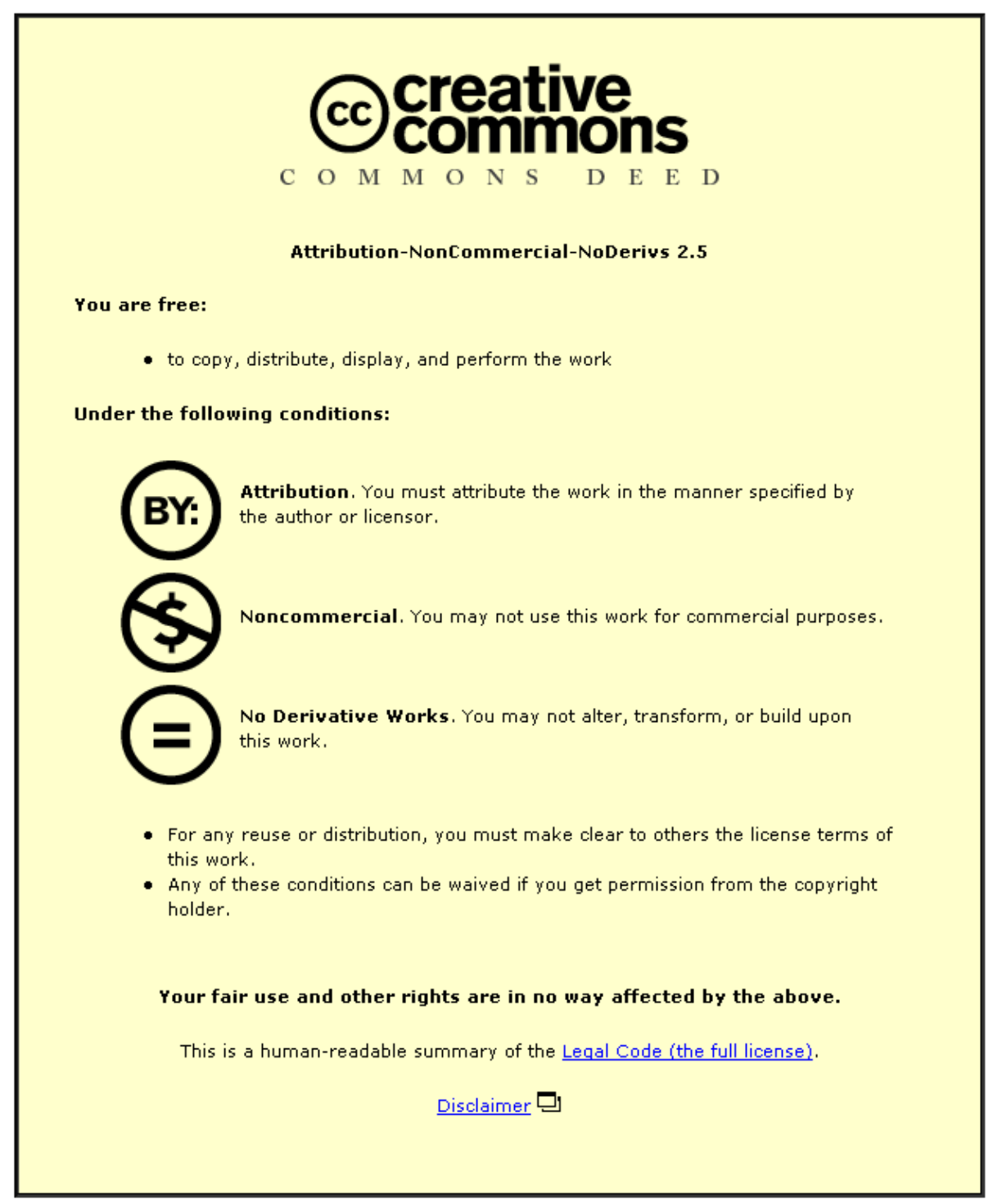

For the full text of this licence, please go to: http://creativecommons.org/licenses/by-nc-nd/2.5/ 


\title{
Hydrodynamic permeability of aggregates of porous particles with an impermeable core
}

Satya Deo ${ }^{1}$, Anatoly Filippov ${ }^{2,3}$, Ashish Tiwari ${ }^{1}$, Sergey Vasin ${ }^{2}$, Victor Starov ${ }^{4}$

${ }^{1}$ Department of Mathematics, University of Allahabad, Allahabad-211002 (U.P.), India

${ }^{2}$ Department of Pure and Applied Mathematics, Moscow State University of Food Production, Volocolamskoye shosse 11, Moscow, 125080, Russia

${ }^{3}$ Department of Mathematics, Gubkin Russian State University of Oil and Gas, Leninsky Prospekt 65, Moscow, 119991, Russia

${ }^{4}$ Department of Chemical Engineering, Loughborough University, Ashby Rd, Loughborough, Leicestershire, LE 11 3TU, UK, V.M.Starov@,lboro.ac.uk

\begin{abstract}
A hydrodynamic permeability of membranes built up by porous cylindrical or spherical particles with impermeable core is investigated. Different versions of a cell method are used to calculate the hydrodynamic permeability of the membranes. Four known boundary conditions, namely, Happel's, Kuwabara's, Kvashnin's and Cunningham/Mehta-Morse's, are considered on the outer surface of the cell. Comparison of the resulting hydrodynamic permeability is undertaken. A possible jump of a shear stress at the fluid-membrane interface, its impact on the hydrodynamic permeability and flow patterns is also investigated. New results related to the calculated hydrodynamic permeability and the theoretical values of Kozeny constant are reported. Both transversal and normal flows of liquid with respect to the cylindrical fibers that compose the membrane are studied. The deduced theoretical results can be applied for the investigation of the hydrodynamic permeability of colloidal cake layers on the membrane surface, the hydrodynamic permeability of woven materials.
\end{abstract}

KEY WORDS: Hydrodynamic permeability, Navier boundary condition, stress jump boundary condition.

*Corresponding author. Tel.: +7 499 1587083, Fax: +7 4991580371

E-mail addresses: a.filippov@mtu-net.ru (Anatoly Filippov), satyadeo@allduniv.ac.in (Satya Deo) 


\section{Contents}

1. Introduction

1.1 Boundary conditions at solid/liquid interfaces

1.2 Boundary conditions at porous/liquid interfaces

2. The cell method

2.1 Boundary conditions at the outer cell surface

2.2 Cell models: spherical particles

2.3 Cell models: cylindrical particles

3. Flow perpendicular to a system of parallel cylinders covered with a porous layer

\subsection{Statement of the problem}

\subsection{Solution of the problem}

3.3. Drag force and hydrodynamic permeability

3.3.1. Hydrodynamic permeability of a porous cylinder-in-cell without impermeable core and with stress jump condition

3.3.2. Hydrodynamic permeability of a porous cylinder-in-cell without impermeable core and with continuity conditions

3.3.3. Hydrodynamic permeability of a membrane built up by solid cylinders

3.4. Results and discussion

4. Flow along parallel cylinders covered with a porous layer

4.1. Set of porous particles

4.2. Set of solid impermeable particles

5. Comparison of different cell models

5.1. Composite particles with a porous layer on the surface

5.2. Completely porous particles

5.3. Rigid impermeable particles

Conclusions

Acknowledgements

List of main symbols

References 


\section{Introduction}

Membrane filtration processes, sedimentation, flow of underground water and oil are only few examples o flows. The basic principle of the cell model is to replace a system of randomly oriented cylindrical particles by a periodic array of spheres or cylinders embedded in a center spherical or cylindrical liquid cells. Appropriate boundary conditions on the cell boundary are supposed to take into account the influence of surrounding particles on the flow inside the cell and the force applied to the particle in the center of the cell.

In the course of filtration processes the structure of the membrane can change due to (i) dissolution of particles, (ii) adsorption of polymers on the surfaces of the particles usually referred to as a poisoning. Both the above mentioned processes result in a formation of a porous shell (in the form of a colloidal layer or a gel layer) on the solid particle's surface, which are usually hard to remove [2]. The presence of porous shell on solid particles has a clear impact on the drag force exerted by the flow on the particles.

Flow through porous shells is frequently modeled by Brinkman's equation [3], which is a modified form of the Darcy's equation. However, it has been observed that the results obtained based on the Brinkman's equations do not agree with the experimental data for non-homogeneous porous media. A modification of the Brinkman's equation was suggested in [4] for the media having non-homogeneous porosity. To overcome this problem it is possible for example to use "variable porosity model" for the liquid/porous boundary region [24] - so-called second Brinkman correction.

We assume below that porous shells under consideration have a uniform porosity.

The membranes under investigation below are supposed to be built by either non-porous particles with a rough surface or particles covered by a porous shell. The latter shells also have a rough surface, and a scale of roughness is equal or even bigger than the average pore size inside the shell. The important problem is a correct selection of boundary conditions on surfaces of nonporous but rough surfaces of particles or porous shell of particles. This problem is discussed below.

\subsection{Boundary conditions at solid/liquid interfaces}

A roughness of the solid-liquid boundary may be significant. The latter is especially important in the case of flow through a porous material or a membrane build up by nanoparticles. In this case the usually adopted no-slip boundary condition can fail and should be replaced by a condition which reflects a presence of roughness of solid-liquid interfaces. Note, the microscopic origin of the no-slip boundary condition has remained elusive and cannot be deduced from the first principles. A slip condition is usually used to account for a roughness of the boundary. The simplest linear slip boundary condition is the Navier boundary condition [5-8]. According to that condition the boundary slip is directly proportional to the tangential shear at the boundary, and the 
proportionality constant is referred to as the slip length. In spherical or cylindrical coordinates the Navier boundary condition is as follows:

$$
\widetilde{\mu}^{(1)} \widetilde{v}_{\theta}^{(1)}=\tilde{l} \widetilde{T}_{\tilde{r} \theta}^{(1)}
$$

where the tilde denotes dimensional values, the superscript (1) means "from the liquid side", $\widetilde{\mu}^{(1)}$ is the dynamic viscosity of the liquid, $\widetilde{v}_{\theta}^{(1)}$ is the slip velocity, the proportionality constant, $\tilde{l}$, is referred to as the slip length, which expresses the ratio of the relative velocity to the tangential viscous stress, $\widetilde{T}_{\widetilde{r} \theta}^{(1)}$. According to (1) the slip velocity is proportional to the shear rate. A linear slip condition was originally introduced by Navier in 1823 [5] and later independently by Maxwell in 1879 [8]. Note, the physical meaning of the slip length, $\tilde{l}$, is to be understood. According to Hocking [9] the latter is $\sim 10^{-6} \mathrm{~cm}$ in the case of smooth surfaces, that is, completely in the range of surface forces. In general, the slip length is determined by the nature of the surface roughness.

Classical analysis of macro-flow past sphere and circular cylinder employs the no-slip boundary condition at their surface, that is, zero relative velocity is assumed between the fluid and solid (see for example [10-11]). Vinogradova [12] pointed out that in most cases the no-slip boundary condition yields the correct results for simple flows, and experiments at macroscopic scales are consistent with the no-slip boundary condition.

However, there exist both experimental and theoretical reasons for relaxing the no-slip hypothesis. An improved understanding of the effect of the fluid-solid interaction on flows over rough solid surfaces has received a considerable attention [13-16]. Molecular dynamics simulations by Qian et. al. [15-16] have reviled a presence of a slippage between the fluid and the solid, in violation of the no-slip boundary condition. These findings cast doubt on the general applicability of the continuum hydrodynamics model of micro- and nanofluidics, where the boundary conditions may have macroscopic implications. Detailed studies of the fluid-solid interaction by Thompson and Troian [14] showed that the degree of slip depends on a number of interfacial parameters including unequal surface walls porosity, fluid densities and the strength of the wall - fluid interaction. All those combined effects may prevent sticking of fluid layer(s) to the solid wall, thus allowing slip to occur. The Navier boundary condition has been validated by Qian and Wang [17] for the driven cavity problem through molecular dynamic and continuum hydrodynamic simulation for a Newtonian fluid, where the no-slip boundary condition causes unphysical stress divergence. The slip length is determined from molecular dynamics simulations and when used as an input for the continuum hydrodynamic simulation. It has been shown by Qian et al. $[15,16]$ that molecular dynamics results can be successfully reproduced in that way. Matthews and Hill [18] proposed that a different postulate, namely the Navier boundary condition, may be a more accurate reflection of physical reality at a fluid/solid interface at micro or nano scales. The no-slip boundary condition is 
in fact an approximation to the Navier boundary condition, corresponding to $\tilde{l}=0$. The limit of infinite slip length corresponds to the conventional condition for a liquid/gas interface (perfect slip) [12]. As the amount of slip is small enough for macroscopic flows, the Navier boundary condition is practically indistinguishable from the no-slip condition in the most cases on the macroscopic scale for smooth surfaces. Richardson [19] has shown that for a general Newtonian fluid flow in the immediate neighborhood of a rough solid wall the no-slip boundary condition is the relevant macroscopic boundary condition, in the sense that deviations between the results for no-slip and infinite slip are of the order of the slip length, which is unknown in the case of rough surfaces. In the case of micro or nano scale problems, the difference between the solutions for no-slip and infinite slip is of an order comparable to the dimensions of the flow, and that is they are of a special interest in micro and nanofluidics studies.

Matthews and Hill in [18] examined also a steady low-Reynolds-number flow around a nanosphere and a circular nanocylinder in a Newtonian fluid; with the no-slip boundary condition replaced by a Navier boundary condition and use the method of matched asymptotic expansions. Their model includes a single parameter to account for the slip, the slip length $\tilde{l}$, which is constant and made dimensionless with respect to the corresponding radius, which is assumed to be of the same order of magnitude as the slip length. Numerical results are presented for the two extreme cases, $\tilde{l}=0$ corresponding to classical no-slop theory, and $\tilde{l}=\infty$ corresponding to the perfect slip. The streamlines for $\tilde{l}>0$ are closer to the body than for $\tilde{l}=0$, while the frictional drag for $\tilde{l}>0$ is reduced below the values for $\tilde{l}=0$, as might be expected. For the circular cylinder, results corresponding to $\tilde{l}=\infty$ are in complete agreement with low-Reynolds-number experimental results, and this excellent agreement is much better than that predicted by the no-slip boundary condition. It is well known that the surface indentations generate reduced drag in the case of a flow around a dimpled sphere (a golf ball). The surface indentations not only make the ball go further but its flight is more predictable than that of an equivalent ball with a smooth surface. Really, the reduced drag is one consequence predicted from the analysis presented by Matthews and Hill [18].

The Navier boundary condition assumes that the degree of slip is independent of the shear rate, that is $\tilde{l}$ is constant. However, molecular dynamic simulations by Thompson and Troian [14] have shown that $\tilde{l}$ is a nonlinear function of the shear rate over a large range of shear rates. At low shear rates $\tilde{l}$ remains constant, but at high shear rates $\tilde{l}$ increases rapidly with the shear rate [14].

Note, above we discussed mostly the slip length for the case of smooth solid surfaces. The situation is far more complicated in the case of rough surfaces, where the slip length is determined by the nature of roughness. 


\subsection{Boundary conditions at porous/liquid interfaces}

In the course of filtration of water soluble polymer/protein solutions the latter can adsorb on the inner membrane surface. Also in the course of membrane filtration the particles/fibers, which the membrane is built of, can partially dissolve. Both latter processes results in a formation of porous layers on the inner membrane surface. Recently Sokhan et. al. [20, 21] have shown that fluid flows in carbon nanopores and nanotubes are characterized by a large slip length. The large values of the slip length probably indirectly show the presence of microporous structures on the solid surface.

In the case of presence of porous shells on the particles/fibers surface the Navier boundary condition is not enough for an adequate description of the flow. It is necessary to consider a flow inside porous layers, which is usually modeled using Brinkman's equation. However, the latter requires specifying boundary conditions at the liquid-porous layer interface because the usually used continuity conditions of both the velocity vector and the stress tensor may not be valid. Note, if the thickness of the porous layer tends to zero the latter conditions result in a no-slip condition. However, it should tend in this case to Navier slip condition.

Let us show that Navier-Stokes equations in a liquid above the porous layer and Brinkman's equation inside the porous layer are not in a contradiction with the jump conditions for the stress tensor on the liquid-porous layer interface. Navier - Stokes equations inside the liquid and Brinkman's equation inside the porous layer can be written as

$$
\begin{aligned}
& \operatorname{div} \widetilde{T}^{(1)}=0, \\
& \operatorname{div} \vec{v}^{(1)}=0
\end{aligned}
$$

and

$$
\begin{aligned}
& \operatorname{div} \widetilde{T}^{(2)}-\frac{\widetilde{\mu}^{(1)}}{\widetilde{k}} \widetilde{\vec{v}^{(2)}}=0, \\
& \operatorname{div} \widetilde{\vec{v}}^{(2)}=0
\end{aligned}
$$

where $\widetilde{T}^{(1)}, \widetilde{T}^{(2)}$ are the corresponding stress tensors; $\tilde{k}$ is the permeability of the porous layer. $\tilde{\mu}^{(1)}$ is the viscosity of the clear fluid, $\widetilde{v}^{(k)}, \quad k=1,2$ are velocity vectors outside and inside the porous cylindrical shell, respectively. Systems of equations (2) and (3) can be rewritten as

$$
\begin{aligned}
& \operatorname{div} \hat{\tilde{T}}^{(1)}=0, \quad \hat{\tilde{T}}^{(1)}=\widetilde{T}^{(1)}+\widetilde{\lambda}^{(1)} \widetilde{I}^{(1)} \\
& \operatorname{div} \vec{v}^{(1)}=0
\end{aligned}
$$

and 
$\operatorname{div} \hat{\tilde{T}}^{(2)}-\frac{\widetilde{\mu}^{(1)}}{\widetilde{k}} \vec{v}^{(2)}=0, \quad \hat{\tilde{T}}^{(2)}=\widetilde{T}^{(2)}+\widetilde{\lambda}^{(2)} \widetilde{I}^{(2)}$

$\operatorname{div} \vec{v}^{(2)}=0$

where $I^{(k)}, k=1,2$ are arbitrary tensors satisfying the condition $\operatorname{div} \widetilde{I}^{(k)}=0, \quad k=1,2, \tilde{\lambda}^{(1)}, \quad \tilde{\lambda}^{(2)}$ are arbitrary constants. Let us select tensors $I^{(k)}, k=1,2$ as follows: $\widetilde{I}_{i j}^{(k)}=v_{j}^{(k)}, \quad k=1,2, \quad i, j=1,2,3$. First equations in systems (4) and (5) after such transformation are valid because of the incompressibility conditions (second equations in systems (4) and (5)). Using exactly the same consideration as in [22] we can show that tangential components of tensors $\hat{\widetilde{T}}^{(1)}, \hat{\widetilde{T}}^{(2)}$ should be continuous at the liquid-porous layer interface. Because tangential velocity components are continuous on the same interface we conclude that

$\widetilde{T}_{\widetilde{r} \theta}{ }^{(2)}-\widetilde{T}_{\widetilde{r} \theta}{ }^{(1)}=\left(\widetilde{\lambda}^{(1)}-\widetilde{\lambda}^{(2)}\right) v_{\theta}{ }^{(2)}$

Note that the latter equation is valid for arbitrary constant $\tilde{\lambda}^{(1)}-\tilde{\lambda}^{(2)}$. However, the latter constant cannot be determined using the previous consideration.

Ochoa-Tapia and Whitaker $[23,24]$ deduced a discontinuity condition of the shearing stress at a liquid-porous layer interface. The derivation of the latter condition, which the authors refer to as "jump momentum boundary condition at a fluid-porous dividing surface" based on a averaging procedure of Navier-Stokes equations, velocity field, pressure and stress tensor over liquid outside the porous layer, the liquid inside the porous layer, and the boundary in between. Ochoa-Tapia and Whitaker [23, 24] defined superficial and intrinsic (actual) velocities of the liquid. Similarly they introduced superficial and intrinsic pressures and stress tensors. «Superficial» is related to averaging procedure over a small volume, which includes both liquid and solid inclusions. «Intrinsic» is referred to the average over the liquid part of the small volume. It is easy to see that the ratio of «superficial» and «intrinsic» values is equal to the porosity. The derivations of "jump momentum boundary condition" has been undertaken in $[23,24]$ in parallel with the derivation of Brinkman's equation. In the case of cylindrical interface the jump boundary condition of intrinsic tangential stress on the liquid-porous layer interface takes the following form [23, 24]:

$\tilde{T}_{\tilde{r} \theta}^{(2)}-\tilde{T}_{\tilde{r} \theta}^{(1)}=\frac{\beta \mu^{(1)}}{\sqrt{\tilde{k}}} \tilde{v}_{\theta}^{(2)}$,

where $\beta$ is the only undetermined dimensionless parameter, which should be of the order magnitude of $\sim 1$ and can be either positive or negative [23, 24]. Eq (7) determines the unknown constant in Eq. (6). In [25] the parameter $\beta$ was determined using experimental data by Beavers and Joseph [26] and a reasonable agreement between the theoretical predictions based on the boundary condition (7) and experimental data has been achieved. 
The authors of ref [25] using the same method as in [23, 24] deduced that the parameter $\beta$ should be negative.

Below we undertake an attempt to estimate the $\beta$ value. Let us consider for that purpose a simplest flow in Hele-Shaw cell with a porous wall (Fig. 1). The flow is in one dimensional and parallel to the moving plate. The flow is described by the following equations Inside the liquid:

$0=\tilde{\mu}^{(1)} \frac{\partial^{2} \widetilde{v}^{(1)}}{\partial \widetilde{z}^{2}}$,

inside the porous medium

$0=\widetilde{\mu}^{(2)} \frac{\partial^{2} \widetilde{v}^{(2)}}{\partial \widetilde{z}^{2}}-\frac{\widetilde{\mu}^{(1)}}{\widetilde{k}} \widetilde{v}^{(2)}$

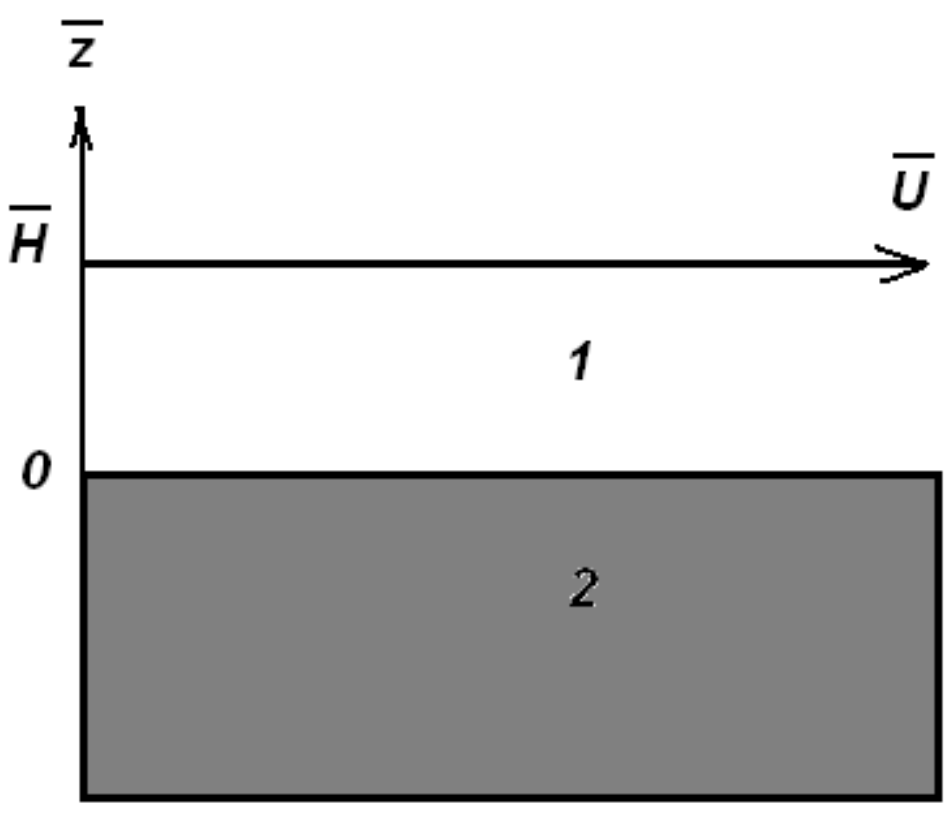

Fig. 1. 1- liquid, 2-porous medium, $\widetilde{U}$-velocity of the upper plate on the distance $\widetilde{H}$ from the porous medium.

With the following boundary conditions

$\widetilde{v}^{(1)}(\widetilde{H})=\widetilde{U}$

$\widetilde{v}^{(1)}(0)=\widetilde{v}^{(2)}(0)$

$\widetilde{v}^{(2)}(-\infty)=0$

$\left.\widetilde{\mu}^{(2)} \frac{\partial \widetilde{v}^{(2)}}{\partial \widetilde{z}}\right|_{\tilde{z}=0}-\left.\widetilde{\mu}^{(1)} \frac{\partial \widetilde{v}^{(1)}}{\partial \widetilde{z}}\right|_{\tilde{z}=0}=\frac{\widetilde{\mu}^{(1)} \beta}{\sqrt{\widetilde{k}}} \widetilde{v}^{(2)}$ 
Let $\widetilde{v}_{0}=\widetilde{v}^{(1)}(0)=\widetilde{v}^{(2)}(0)$ be an unknown velocity to be determined at the liquid-porous layer interface. Then solutions of the Eqs $(8,9)$ with boundary conditions (10-12) are $\widetilde{v}^{(1)}(\widetilde{z})=\frac{\widetilde{z}}{\widetilde{H}}\left(\widetilde{U}-\widetilde{v}_{0}\right)+\widetilde{v}_{0}, \quad \widetilde{z}>0$

$$
\tilde{v}^{(2)}(\tilde{z})=\tilde{v}_{0} \exp \left(\frac{\tilde{z}}{\lambda \sqrt{\tilde{k}}}\right), \tilde{z}<0
$$

where $\lambda=\sqrt{\frac{\widetilde{\mu}^{(2)}}{\widetilde{\mu}^{(1)}}}$.

Solutions (14) and (15) should be substituted into boundary condition (13). The latter results in the following equation for the unknown velocity at the interface:

$$
\widetilde{v}_{0}=\frac{\tilde{U}}{1+\frac{\widetilde{H}}{\sqrt{\tilde{k}}}(\lambda-\beta)}
$$

It is obvious that the velocity at the liquid-porous layer interface cannot become higher than $\tilde{U}$, Hence, according to Eq (16) we conclude that $\beta$ should satisfy the following inequality: $\beta<\lambda$.

Let us introduce the velocity in the absence of tensor jump boundary condition: $\widetilde{v}_{00}=\frac{\widetilde{U}}{1+\frac{\widetilde{H}}{\sqrt{\widetilde{k}}} \lambda}$.

Based on Eq. (16) we can conclude: if $0<\beta<\lambda$ then according to (16) $\widetilde{v}_{0}>\widetilde{v}_{00}$, however, if $\beta<0$, then $\widetilde{v}_{0}<\widetilde{v}_{00}$. If $\beta \rightarrow-\infty$ then $\widetilde{v}_{0} \rightarrow 0$. The latter means, that $\beta \rightarrow-\infty$ corresponds to no-slip boundary condition on the liquid-porous layer interface. So, we have evaluated the range of possible values of $\beta$ as: $-\infty<\beta<\lambda$. Below we use the boundary conditions in the form presented by Eq (7).

\section{The cell method}

\subsection{Boundary conditions at the outer cell surface}

Flow through permeable aggregates of particles is frequently encountered at investigation of membrane filtration with cake (gel) layer formation [27, 28], sedimentation, flow of underground water, oil etc. The cell model [1] has been very effectively used for investigation of such flows. The basic principle of the cell model is as follows: a system of randomly oriented cylindrical particles is replaced by a periodic array of spheres or cylinders embedded in identical spherical or cylindrical liquid cells. Appropriate boundary conditions on the outer cell boundary are supposed to take into account the effect of surrounding particles on the particle in the center of the cell.

Authors of [29] revised the cell model method for addressing hydrodynamic flow through system of solid particles. The starting point of the analysis is the general problem formulation intended for describing a pressure driven flow through a membrane, which was considered as a set 
of representative cells having arbitrary shape and containing any number of particles. Using the general problem formulation, the hydrodynamic field inside an individual representative cell is related to the applied pressure difference and the external flow velocity. Relationships containing integrals over the outer boundary of a representative cell has been derived in [29]. Assuming that the representative cell is a sphere containing a single particle in the centre, the derived general relationships were transformed into outer cell boundary conditions employed in the literature by different authors. Choosing different sets of the outer boundary conditions, different models were considered and compared with each other and with the results obtained by others for regular particle arrays. The common and different features of the hydrodynamic and electrodynamic versions of the cell model approaches were analyzed. Finally, it was discussed, which version of the cell model gives the best approximation, while describing pressure and electrically driven flows through a membrane and sedimentation of particles. Authors of [29] concluded that the model based on the Kuwabara's boundary condition gives a better approximation than the Cunningham/Mehta-Morse's and Happel's models. Kvashnin's model was not considered in [29]. According to [29] the best versions of the spherical cell approximation are valid for purely pressure driven flow as well as in the presence of a body force of arbitrary origin provided that such a body force can be represented as a gradient of a scalar function of coordinates. The latter conclusions were illustrated by two examples: the sedimentation and the electroosmotic flow for the case of strong overlap of the double layers. For the Smoluchowski limit of the electroosmotic velocity, using the Kuwabara condition leads to the classical Smoluchowski exact result which is independent of the volume fraction. The predictions based on Cunningham/Mehta-Morse's and Happel's models depend on the volume fraction and coincide with the Smoluchowski result for infinite dilution. The conducted analysis of two limiting cases for electroosmotic velocity shows that the Kuwabara boundary condition should be recommended for addressing electroosmosis. For the strong overlap of the double layers, similarly to the case of pressure driven flow, the Kuwabara condition gives the best approximation compared to other models [29].

The presence of porous shell on solid particles inside the membranes or other aggregates has a clear impact on the drag force exerted by the fluid on the particles.

Happel [30] and Kuwabara [31] proposed cell models in which both particle and outer envelope are spheres/cylinders. The advantage of such formulation is that it leads to an axially symmetric flow that has a simple analytical solution in a closed form. According to the Happel's model nil shear stress is assumed on the outer cell boundary, while according to the Kuwabara's model nil vorticity is assumed on the outer cell boundary. Both models results in similar velocity fields and drag forces. The Happel's model does not require an exchange of mechanical energy between the cell and the environment, while the Kuwabara's model requires an exchange of 
mechanical energy with the environment. Two other cell models have been suggested by Kvashnin [32] and Cunningham [33] (and later by Mehta-Morse [34]) using different boundary conditions on the outer boundary of the cell. Kvashnin [32] proposed the condition that the tangential component of velocity reaches a minimum at the cell surface with respect to radial distance, signifying the symmetry on the cell. Cunningham [33] and Mehta-Morse [34] assumed the tangential velocity as a component of the average fluid velocity, signifying the homogeneity of the flow on the cell boundary. The importance of the latter boundary condition [33-34] is as follows: we are interested in the flow behavior on a large scale, hence, we should average the flow variables on a small scale over a cell volume to obtain the large scale behavior. It is precisely, what model [33-34] states.

\subsection{Cell models: spherical particles}

Probably [35] was the first publication on the application of the cell model and Brinkman's equations for investigations of permeability of membranes built up by porous particles. A flow around a spherical porous particles located in a centre of spherical cell with Cunningham/MehtaMorse's boundary condition was considered in [35]. Solutions were given for two cases: identical liquids inside and outside the particle and non-mixing liquids inside and outside the particles [35]. An exact expression for the force applied to the porous particles was calculated in both cases. In [36] the problem of constant-velocity motion in an unbounded incompressible viscous fluid was solved for a spherical particle consisting of a rigid core, covered with a uniform porous layer not deformable by hydrodynamic flow. A finite algebraic formula was obtained for determining the force that acts on a particle in the general case of different liquid viscosities inside and outside the porous layer. In [37] the problem of a uniform flow (Cunningham/Mehta-Morse's model) of a viscous incompressible liquid flow around a porous spherical particle inside a liquid spherical cell was considered. An expression for the hydrodynamic permeability of a membrane built up by porous particles was deduced. In development of [37] a problem of uniform flow of viscous incompressible liquid around a rigid particle covered with a porous layer and located inside the spherical cell was solved at small Reynolds numbers [38]. Based on the cell method, the hydrodynamic permeability of the membrane built up by porous particles with rigid impermeable cores was also calculated in [38].

Filippov et al. [39] reviewed investigations on the hydrodynamic permeability of porous membranes built up by completely porous particles or particles with a porous layer using Cunningham/Mehta-Morse's boundary condition. The influence of a porous shell on the total permeability was discussed. It was shown [39] that the fluid flow penetrates only on a finite depth inside the porous shell of the particles. Different limiting cases were investigated in [39], which allowed to recover previously known results. Vasin et al. [40] used the cell method to model permeability of membrane built up by solid spherical particles with a permeable porous shell and 
reviewed all the four above mentioned boundary conditions. A flow around an isolated spherical particle with a porous shell was considered also and a number of limiting cases were discussed and compared with the corresponding results obtained earlier [40].

Dassios et al. [41] found a solution of Stokes equation in spheroidal coordinates and used it for a Stokes flow in spheroidal particle-in-cell models [42].

\subsection{Cell models: cylindrical particles}

Vasin and Filippov [43] used all four versions of the cell method to evaluate the hydrodynamic permeability of membrane built up by porous cylindrical particles with impermeable cores (fibrous membranes). Theoretical predictions and experimental results were compared, various limiting cases were deduced and compared with known literature data.

Stechkina [44] evaluated the drag force experienced by porous cylinders in a viscous fluid flow at low Reynolds number using collocation method. Pop and Cheng [45] reported an analytical study of the steady incompressible flow around a circular cylinder embedded in a constant porosity medium based on the Brinkman model, proving that separation of the flow does not take place at the surface of the cylinder and also explained the velocity overshoot behaviour. Stokes flow past a swarm of porous circular cylinders with Happel's and Kuwabara's boundary conditions was discussed by Deo [46]. The flow around nanospheres and nanocylinders was investigated by Mathews and Hill [18] and they employed a boundary condition that attempts to account for boundary slip due to the tangential shear at the boundary using a slip length parameter. Palaniappan et al. [47] studied the two dimensional Stokes flow around permeable cylinders. Datta and Shukla [48] calculated the drag on a cylinder, using slip boundary condition and also deduced that the slippage on the cylinder reduces the drag. Deo and Yadav [49] studied the problem of Stokes flow through a swarm of porous circular cylinder-in-cell enclosing an impermeable core with Kuwabara's boundary condition.

The hydrodynamic permeability of a membrane built up by a set of identical impermeable cylinders covered with a porous layer was calculated in [50] using the cell method. Both transverse and longitudinal flows of filtering liquid with respect to the cylindrical fibers that compose the membrane were studied. Boundary conditions on the cell surface that correspond to the Happel's, Kuwabara's, Kvashnin's, and Cunningham/Mehta-Morse's models were considered. Theoretical values of Kozeny constants were calculated.

The stream function for a slow viscous flow through an array of porous cylindrical particles with Happel's boundary condition was considered in [51]. The drag force exerted to each porous cylindrical particle in a cell was evaluated.

A number of authors have used the above-mentioned stress jump boundary condition [2324] at liquid/porous layer interface in various flow problems. Srivastava and Srivastava [52] studied 
the Stokes flow around a porous sphere using stress jump condition at the liquid-porous layer interface and concluded that drag on porous sphere decreases with increase of permeability of the medium. Recently, Bhattacharya and Raja Sekhar [53] have used the same jump boundary conditions to discuss Stokes flows inside a porous spherical shell.

\section{Flow perpendicular to a system of parallel cylinders covered with a porous layer}

Below we present a consideration of the Stokes flow through a membrane built up by porous circular cylinders with an impermeable core with stress jump boundary condition at the liquid-porous interface using the four known boundary conditions applied on the cell surface: Happel's, Kuwabara's, Kvashnin's and Cunningham/Mehta-Morse's boundary conditions. Some previous results published for cell models are been recovered/verified below. A comparative study of all the four boundary conditions shows some interesting results for the hydrodynamic permeability through the porous media.

\subsection{Statement of the problem}

Below we consider an axi-symmetric Stokes flow of a viscous incompressible fluid through a swarm (membrane) of porous cylindrical particles of radius $\tilde{b}$ with an impermeable core of radius $\tilde{a}$ (Fig. 2). The above model is equivalent to a co-axial porous cylindrical shell enclosing the impermeable core. Further, we assume that, this porous shell is enveloped by a concentric cylinder of radius $\tilde{c}(\tilde{c}>\tilde{b})$, which is the cell outer surface. The Stokes flow of a Newtonian fluid with dynamic viscosity $\widetilde{\mu}^{(1)}$ is assumed to be steady and axi-symmetric. We assume that the fluid approaches the cell surface and partially passes through the composite cylinder perpendicular to the axis of the cylinder ( $\tilde{z}$-axis) with velocity $\tilde{\mathbf{U}}(|\tilde{\mathbf{U}}|=\tilde{U})$ from left to right. The radius $\tilde{c}$ of the cell is chosen in such a way that the particle volume fraction, $\gamma$, of the porous cylinders is equal to the particle volume fraction inside the cell:

$$
\gamma=\pi \tilde{b}^{2} / \pi \tilde{c}^{2}=1-\varepsilon
$$

where $\varepsilon$ is the porosity of the porous medium.

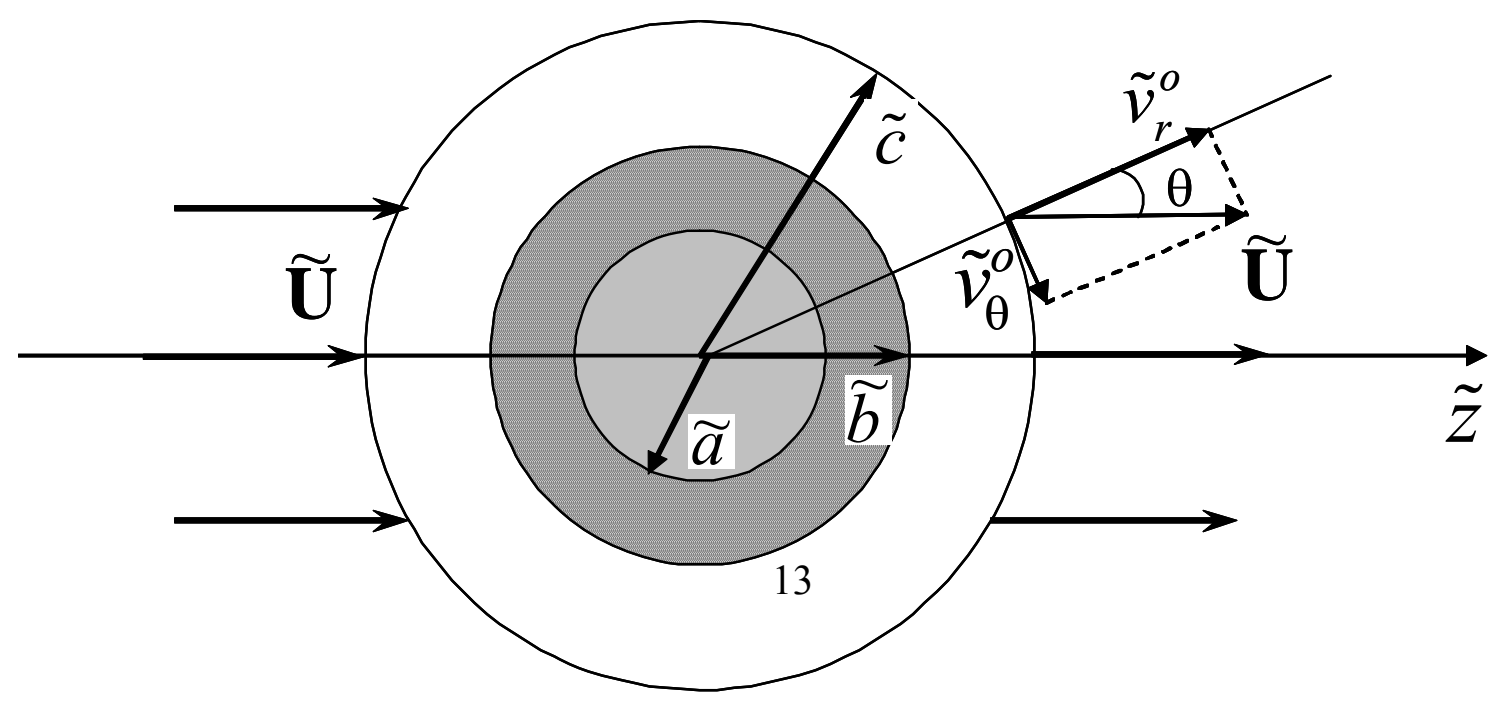


Fig. 2. Schematic presentation of a cylindrical cell of radius $\widetilde{c}$ with solid impermeable particle of radius $\tilde{a}$ covered with porous layer ( $\widetilde{b}$ is a combined radius of the particle with the porous shell) placed in a transverse flow of a liquid from left to right.

The governing equations for the creeping flow of an incompressible Newtonian fluid, which lies in the region outside the porous cylindrical shell is governed by Stokes equations [1] as

$$
\tilde{\mu}^{(1)} \tilde{\nabla}^{2} \tilde{\mathbf{v}}^{(1)}=\nabla \tilde{p}^{(1)} .
$$

The flow inside the porous cylindrical shell is governed by the Brinkman's equation [3]:

$$
\tilde{\mu}^{(2)} \tilde{\nabla}^{2} \tilde{\mathbf{v}}^{(2)}-\left(\tilde{\mu}^{(1)} / \tilde{k}\right) \tilde{\mathbf{v}}^{(2)}=\tilde{\nabla} \tilde{p}^{(2)},
$$

superscripts (1) and (2) refer to the external zone and the porous layer, respectively; $\tilde{\mu}^{(1)}$ is the viscosity of the clear fluid, $\tilde{\mu}^{(2)}$ denotes the effective viscosity inside the porous layer; $\tilde{k}$ is the permeability of the porous layer; $\tilde{\mathbf{v}}^{(i)}, \tilde{p}^{(i)}, i=1,2$ are the velocity vector and pressure outside and inside the porous cylindrical shell, respectively. The viscosities $\tilde{\mu}^{(1)}$ and $\tilde{\mu}^{(2)}$ are, in general, different. Eq (19) reduces to the Stokes equation for large permeability $\tilde{k}\left(\tilde{\mu}^{(2)} \tilde{\nabla}^{2} \tilde{\mathbf{v}}^{(2)}=\tilde{\nabla} \tilde{p}^{(2)}\right)$ however, for low permeability this equation transforms into Darcy's equation $\left(-\left(\tilde{\mu}^{(1)} / \tilde{k}\right) \tilde{\mathbf{v}}^{(2)}=\tilde{\nabla} \tilde{p}^{(2)}\right)$.

The continuity equations for incompressible fluids must be satisfied in both regions:

$$
\tilde{\nabla} \cdot \tilde{\mathbf{v}}^{(i)}=0, \quad i=1,2 .
$$

The cylindrical system of coordinates $\tilde{r}, \theta, \tilde{z}$, with the origin in the center of the cylindrical particle, $\tilde{z}$ axis oriented along the cylinder and the polar axis directed along the flow is used below (Fig. 2).

Let us introduce dimensionless variables and constants as follows:

$$
\begin{aligned}
& \gamma=\frac{\widetilde{b}^{2}}{\widetilde{c}^{2}}, m=\frac{\widetilde{c}}{\widetilde{b}}=\frac{1}{\sqrt{\gamma}}, l=\frac{\widetilde{a}}{\widetilde{b}}, r=\frac{\widetilde{r}}{\widetilde{b}}, \nabla=\widetilde{\nabla} \cdot \widetilde{b}, \\
& v=\frac{\widetilde{v}}{\widetilde{U}}, p=\frac{\widetilde{p}}{\widetilde{p}_{0}}, \widetilde{p}_{0}=\frac{\widetilde{U} \widetilde{\mu}^{(1)}}{\widetilde{b}}, \lambda=\sqrt{\frac{\widetilde{\mu}^{(2)}}{\widetilde{\mu}^{(1)}}}, \sigma=\frac{\widetilde{b}}{\sqrt{\widetilde{k}}}, \alpha=\frac{\sigma}{\lambda}
\end{aligned}
$$

The equations of continuity (20) for axi-symmetric, incompressible viscous fluid in cylindrical coordinates in dimensionless form in both regions can be rewritten as

$$
\frac{\partial}{\partial r}\left(r v_{r}^{(i)}\right)+\frac{\partial}{\partial \theta}\left(v_{\theta}^{(i)}\right)=0
$$


where, $v_{r}^{(i)}$ and $v_{\theta}^{(i)}, i=1,2$ are components of velocities in the direction of $r$ and $\theta$, respectively.

The stream functions $\psi^{(i)}(r, \theta)$ in both regions satisfying continuity equations (22) are defined as

$$
v_{r}^{(i)}=\frac{1}{r} \frac{\partial \psi^{(i)}}{\partial \theta} ; \quad v_{\theta}^{(i)}=-\frac{\partial \psi^{(i)}}{\partial r}, \quad i=1,2 .
$$

Using stream functions and eliminating the pressures from both Eqs (18) and (19) and using definitions (23) we arrive to the following fourth order partial differential equations for stream functions:

$$
\begin{aligned}
& \nabla^{2}\left(\nabla^{2} \psi^{(1)}\right)=0, \\
& \nabla^{2}\left(\nabla^{2}-\alpha^{2}\right) \psi^{(2)}=0,
\end{aligned}
$$

where

$$
\nabla^{2}=\frac{\partial^{2}}{\partial r^{2}}+\frac{1}{r} \frac{\partial}{\partial r}+\frac{1}{r^{2}} \frac{\partial^{2}}{\partial \theta^{2}}
$$

The pressure can be obtained in both regions by integrating the following relations [1]:

$$
\begin{aligned}
& \frac{\partial p^{(1)}}{\partial r}=\nabla^{2} v_{r}^{(1)}-\frac{v_{r}^{(1)}}{r^{2}}-\frac{2}{r^{2}} \frac{\partial v_{\theta}^{(1)}}{\partial \theta}, \\
& \frac{1}{r} \frac{\partial p^{(1)}}{\partial \theta}=\nabla^{2} v_{\theta}^{(1)}-\frac{v_{\theta}^{(1)}}{r^{2}}+\frac{2}{r^{2}} \frac{\partial v_{r}^{(1)}}{\partial \theta}, \\
& \frac{\partial p^{(2)}}{\partial r}=\lambda^{2}\left(\nabla^{2} v_{r}^{(2)}-\frac{v_{r}^{(2)}}{r^{2}}-\frac{2}{r^{2}} \frac{\partial v_{\theta}^{(2)}}{\partial \theta}\right)-\sigma^{2} v_{r}^{(2)}, \\
& \frac{1}{r} \frac{\partial p^{(2)}}{\partial \theta}=\lambda^{2}\left(\nabla^{2} v_{\theta}^{(2)}-\frac{v_{\theta}^{(2)}}{r^{2}}+\frac{2}{r^{2}} \frac{\partial v_{r}^{(2)}}{\partial \theta}\right)-\sigma^{2} v_{\theta}^{(2)} .
\end{aligned}
$$

A solution of the Stokes Eq (24) can be expressed as

$$
\psi^{(1)}(r, \theta)=\left[A_{1} r+B_{1} r^{3}+C_{1} / r+D_{1} r \ln r\right] \sin \theta .
$$

A solution of the Brinkman Eq (25) can be written as

$$
\psi^{(2)}(r, \theta)=\left[A_{2} r+B_{2} / r+C_{2} I_{1}(\alpha r)+D_{2} K_{1}(\alpha r)\right] \sin \theta .
$$

Here, $I_{1}(\alpha r)$ and $K_{1}(\alpha r)$ are the modified Bessel's functions of the order one of the first and second kinds (Abramowitz and Stegun [55]), respectively.

Substituting expressions (31)-(32) into Eq. (23), (27)-(30) we arrive at

$$
\begin{aligned}
& v_{r}^{(1)}=\left[A_{1}+B_{1} r^{2}+\frac{C_{1}}{r^{2}}+D_{1} \ln r\right] \cos \theta, \\
& v_{\theta}^{(1)}=\left[-A_{1}-3 B_{1} r^{2}+\frac{C_{1}}{r^{2}}-D_{1}(1+\ln r)\right] \sin \theta,
\end{aligned}
$$




$$
\begin{aligned}
& p^{(1)}=\left[8 B_{1} r-\frac{2 D_{1}}{r}\right] \cos \theta, \\
& v_{r}^{(2)}=\left[A_{2}+\frac{B_{2}}{r^{2}}+\frac{C_{2}}{r} I_{1}(\alpha r)+\frac{D_{2}}{r} K_{1}(\alpha r)\right] \cos \theta, \\
& v_{\theta}^{(2)}=\left[-A_{2}+\frac{B_{2}}{r^{2}}-\frac{C_{2} s}{2}\left(I_{0}(\alpha r)+I_{2}(\alpha r)\right)+\frac{D_{2} s}{2}\left(K_{0}(\alpha r)+K_{2}(\alpha r)\right)\right] \sin \theta, \\
& p^{(2)}=\lambda^{2} \alpha^{2}\left(-A_{2} r+\frac{B_{2}}{r}\right) \cos \theta .
\end{aligned}
$$

\subsection{Solution of the problem}

The dimensionless boundary conditions for the problem under investigation are as follows.

Impermeability and no-slip (sticking) boundary conditions are used on the surface of the solid cylindrical core of radius $r=l(\tilde{r}=\tilde{a})$ :

$$
v_{r}^{(2)}(l, \theta)=0, \quad v_{\theta}^{(2)}(l, \theta)=0 .
$$

The continuity of the normal and tangential components of velocity and normal component of stress tensor $T_{r r}^{(i)}$ and jump condition $[23,24]$ for shearing stress $T_{r \theta}^{(i)}$ are employed at the fluidporous layer interface at $r=1(\tilde{r}=\tilde{b})$ :

$$
\begin{aligned}
& v_{r}^{(2)}(1, \theta)=v_{r}^{(1)}(1, \theta), v_{\theta}^{(2)}(1, \theta)=v_{\theta}^{(1)}(1, \theta), \\
& T_{r r}^{(2)}(1, \theta)=T_{r r}^{(1)}(1, \theta), \\
& T_{r \theta}^{(2)}(1, \theta)-T_{r \theta}^{(1)}(1, \theta)=\beta \sigma v_{\theta}^{(2)}(1, \theta),
\end{aligned}
$$

where

$$
\begin{aligned}
& T_{r r}^{(1)}=-p^{(1)}+\frac{2}{r}\left(\frac{\partial^{2} \psi^{(1)}}{\partial r \partial \theta}-\frac{1}{r} \frac{\partial \psi^{(1)}}{\partial \theta}\right), \\
& T_{r \theta}^{(1)}=\frac{1}{r^{2}} \frac{\partial^{2} \psi^{(1)}}{\partial \theta^{2}}+\frac{1}{r} \frac{\partial \psi^{(1)}}{\partial r}-\frac{\partial^{2} \psi^{(1)}}{\partial r^{2}}, \\
& T_{r r}^{(2)}=-p^{(2)}+\frac{2 \lambda^{2}}{r}\left(\frac{\partial^{2} \psi^{(2)}}{\partial r \partial \theta}-\frac{1}{r} \frac{\partial \psi^{(2)}}{\partial \theta}\right), \\
& T_{r \theta}^{(2)}=\lambda^{2}\left(\frac{1}{r^{2}} \frac{\partial^{2} \psi^{(2)}}{\partial \theta^{2}}+\frac{1}{r} \frac{\partial \psi^{(2)}}{\partial r}-\frac{\partial^{2} \psi^{(2)}}{\partial r^{2}}\right) .
\end{aligned}
$$


Condition (42) includes dimensionless jump coefficient of the order of one and sign of $\beta$ may either be positive or negative [24]. The jump coefficient $\beta$ signifies the difference in the shear stress at the fluid-porous layer interface and assumes values between approximately -1 and 1 .

Special attention is usually paid to the boundary conditions on the outer cell boundary, $r=m(\tilde{r}=\tilde{c})$. There are four frequently used versions of those boundary conditions, which are referred below as Happel's, Kuwabara's, Kvashnin's and Cunningham/Mehta-Morse's models. All four models assume continuity of the radial component of the liquid velocity on the outer cell surface $(r=m(\tilde{r}=\tilde{c}))$ :

$$
v_{r}^{(1)}(m, \theta)=\cos \theta \text {. }
$$

Let us consider an additional condition used in each of the mentioned models. According to the Happel's model [30] the tangential viscous stress vanishes on the cell boundary $(r=m(\tilde{r}=\tilde{c})):$

$$
T_{r \theta}^{(1)}(m, \theta)=0 .
$$

According to Kuwabara's model [31] the curl vanishes on the cell boundary $(r=m(\tilde{r}=\tilde{c}))$, that is the flow is assumed to be potential one:

$$
\nabla^{2} \psi^{(1)}(m, \theta)=0 .
$$

According to Kvashnin's model [32] a symmetry condition is introduced as follows:

$$
\frac{\partial v_{\theta}^{(1)}}{\partial r}=0 . \quad(r=m(\tilde{r}=\tilde{c}))
$$

Acknowledging the importance of using unknown velocity as a boundary condition at the cell surface $r=m(\tilde{r}=\tilde{c})$, initially Cunningham [33] and afterwards Mehta and Morse [34] assumed

$$
v_{\theta}^{(1)}(m, \theta)=-\sin \theta
$$

Solving the above problem for given boundary conditions (39)-(47) and one condition from (48)-(51) we can determined all the unknown constants. Expressions for those constants are not given below.

\subsection{Drag force and hydrodynamic permeability}

Integrating the normal and tangential stresses over the surface of the porous cylindrical shell

of radius $\tilde{b}$ in the cell, yields the drag force experienced per unit length $\tilde{F}$ of a cylinder as given below:

$$
\tilde{F}=\int_{0}^{2 \pi}\left(\tilde{T}_{\tilde{r} \tilde{r}}^{(1)} \cos \theta-\tilde{T}_{\tilde{r} \theta}^{(1)} \sin \theta\right)_{\tilde{r}=\tilde{b}} \tilde{b} d \theta=F \tilde{\mu}^{(1)} \tilde{U},
$$


or

$$
F=\int_{0}^{2 \pi}\left(T_{r r}^{(1)} \cos \theta-T_{r \theta}^{(1)} \sin \theta\right)_{r=1} d \theta
$$

is the dimensionless force.

Stress components according to Eqs (43) and (44) are

$$
\begin{aligned}
& T_{r r}^{(1)}=-4\left[r B_{1}+\frac{1}{r^{3}} C_{1}-\frac{1}{r} D_{1}\right] \cos \theta, \\
& T_{r \theta}^{(1)}=-4\left[r B_{1}+\frac{1}{r^{3}} C_{1}\right] \sin \theta .
\end{aligned}
$$

Substitution of those expressions (54) and (55) in (53) we get after integration:

$$
F=4 \pi D_{1} \text {. }
$$

Hydrodynamic permeability of a membrane, $\widetilde{L}_{11}$, built up by those cylinders is defined as the ratio of the uniform flow rate $\tilde{U}$ to the cell gradient pressure $\tilde{F} / \tilde{V}$ [1]:

$$
\tilde{L}_{11}=\frac{\tilde{U}}{\tilde{F} / \tilde{V}}
$$

where, $\tilde{V}=\pi \tilde{c}^{2}$ is the volume of the cell of the unit length.

Substitution the value of $\tilde{F}$ from equation (52) and the value of $\tilde{V}$ from above in equation (57), we get

$$
\tilde{L}_{11}=\frac{\tilde{b}^{2}}{4 \tilde{\mu}^{(1)} \gamma D_{1}}=L_{11} \frac{\tilde{b}^{2}}{\tilde{\mu}^{(1)}},
$$

where,

$$
L_{11}=\frac{1}{4 \gamma D_{1}},
$$

is the dimensionless hydrodynamic permeability of the membrane. The hydrodynamic permeability is a function of five dimensionless parameters, i.e. $L_{11}(l, \sigma, \gamma, \lambda, \beta)$.

3.3.1. Hydrodynamic permeability of a porous cylinder-in-cell without impermeable core and with stress jump condition

In a particular case of $(l=0)$, i.e. no impermeable core within the porous cylinder, we have the following expressions for hydrodynamic permeability.

For Happel's model:

$$
\begin{aligned}
L_{11}= & \Delta_{H}^{\prime} /\left(2 \alpha \lambda \left(\left(\alpha \beta \lambda^{2}-\alpha^{2} \lambda^{3}\right)\left(1+m^{4}\right) I_{1}(\alpha)-2 \beta\left(1-m^{4}\right) I_{2}(\alpha)+2 \alpha \lambda\left(1-m^{4}\right) I_{2}(\alpha)\right.\right. \\
& \left.\left.-2 \beta \lambda^{2}\left(1+m^{4}\right) I_{2}(\alpha)+\alpha^{2} \beta \lambda^{2}\left(1+m^{4}\right) I_{2}(\alpha)+2 \alpha \lambda^{3}\left(1+m^{4}\right) I_{2}(\alpha)\right)\right) /(4 \gamma),
\end{aligned}
$$

where 


$$
\begin{aligned}
\Delta_{H}^{\prime} & =\left(-8 \alpha \lambda^{2}\left(1+m^{4}\right) I_{1}(\alpha)+\alpha^{2} \beta \lambda^{3}\left(1-m^{4}\right) I_{1}(\alpha)-\alpha^{3} \lambda^{4}\left(1-m^{4}\right) I_{1}(\alpha)+16\left(1-m^{4}\right) I_{2}(\alpha)\right. \\
& +16 \alpha \beta \lambda I_{2}(\alpha)+16 \lambda^{2}\left(1+m^{4}\right) I_{2}(\alpha)-2 \alpha \beta \lambda^{3}\left(1-m^{4}\right) I_{2}(\alpha)+\alpha^{3} \beta \lambda^{3}\left(1-m^{4}\right) I_{2}(\alpha) \\
& +2 \alpha^{2} \lambda^{4}\left(1-m^{4}\right) I_{2}(\alpha)+2 \alpha^{2} \beta \lambda^{3}\left(1+m^{4}\right) I_{1}(\alpha) \ln m-2 \alpha^{3} \lambda^{4}\left(1+m^{4}\right) I_{1}(\alpha) \ln m \\
& -\left\{4 \alpha \beta \lambda\left(1-m^{4}\right)-4 \alpha^{2} \lambda^{2}\left(1-m^{4}\right)+4 \alpha \beta \lambda^{3}\left(1+m^{4}\right)-2 \alpha^{3} \beta \lambda^{3}\left(1+m^{4}\right)\right. \\
& \left.\left.-4 \alpha^{2} \lambda^{4}\left(1+m^{4}\right)\right\} I_{2}(\alpha) \ln m\right) .
\end{aligned}
$$

For Kuwabara's model:

$$
\begin{aligned}
L_{11}= & \Delta_{K}^{\prime} /\left(4 m ^ { 4 } \alpha \lambda \left(\left(\alpha \beta \lambda^{2}-\alpha^{2} \lambda^{3}\right) I_{1}(\alpha)+2 \beta I_{2}(\alpha)-2 \alpha \lambda I_{2}(\alpha)\right.\right. \\
& \left.\left.-2 \beta \lambda^{2} I_{2}(\alpha)+\alpha^{2} \beta \lambda^{2} I_{2}(\alpha)+2 \alpha \lambda^{3} I_{2}(\alpha)\right)\right) /(4 \gamma),
\end{aligned}
$$

where

$$
\begin{aligned}
\Delta_{K}^{\prime} & =\left(-16 \alpha \lambda^{2} m^{4} I_{1}(\alpha)-\alpha^{2} \beta \lambda^{3}\left(1-4 m^{2}+3 m^{4}\right) I_{1}(\alpha)-32 m^{4}\left(1-\lambda^{2}\right) I_{2}(\alpha)+\alpha^{3} \lambda^{4}\right. \\
& \left(1-4 m^{2}+3 m^{4}\right) I_{1}(\alpha)+2 \alpha \beta \lambda\left(1+8 m^{2}-m^{4}\right) I_{2}(\alpha)+2 \alpha^{2} \lambda^{2}\left(1+m^{4}\right) I_{2}(\alpha)+2 \alpha \beta \lambda^{3} \\
& \left(1-4 m^{2}+3 m^{4}\right) I_{2}(\alpha)-\alpha^{3} \beta \lambda^{3}\left(1-4 m^{2}+3 m^{4}\right) I_{2}(\alpha)-2 \alpha^{2} \lambda^{4}\left(1-4 m^{2}+3 m^{4}\right) I_{2}(\alpha) \\
& +4 \alpha^{2} \beta \lambda^{3} m^{4} I_{1}(\alpha) \ln m-4 m^{4} \alpha^{3} \lambda^{4} I_{1}(\alpha) \ln m+8 m^{4} \alpha \beta \lambda I_{2}(\alpha) \ln m-8 m^{4} \alpha^{2} \lambda^{2} I_{2}(\alpha) \ln m \\
& \left.-8 m^{4} \alpha \beta \lambda^{3} I_{2}(\alpha) \ln m+4 m^{4} \alpha^{3} \beta \lambda^{3} I_{2}(\alpha) \ln m+8 m^{4} \alpha^{2} \lambda^{4} I_{2}(\alpha) \ln m\right) .
\end{aligned}
$$

For Kvashnin's model:

$$
\begin{aligned}
L_{11} & =\Delta_{K V} / \alpha \lambda\left(\alpha \beta \lambda^{2} I_{1}(\alpha)+3 m^{4} \alpha \beta \lambda^{2} I_{1}(\alpha)-\alpha^{2} \lambda^{3} I_{1}(\alpha)-3 m^{4} \alpha^{2} \lambda^{3} I_{1}(\alpha)-2 \beta I_{2}(\alpha)\right. \\
& +6 m^{4} \beta I_{2}(\alpha)+2 \alpha \lambda I_{2}(\alpha)-6 m^{4} \alpha \lambda I_{2}(\alpha)-2 \beta \lambda^{2} I_{2}(\alpha)-6 m^{4} \beta \lambda^{2} I_{2}(\alpha)+\alpha^{2} \beta \lambda^{2} I_{2}(\alpha) \\
& \left.+3 m^{4} \alpha^{2} \beta \lambda^{2} I_{2}(\alpha)+2 \alpha \lambda^{3} I_{2}(\alpha)+6 m^{4} \alpha \lambda^{3} I_{2}(\alpha)\right) /(4 \gamma),
\end{aligned}
$$

where

$\Delta_{K V}=-4 \alpha \lambda^{2} I_{1}(\alpha)-12 m^{4} \alpha \lambda^{2} I_{1}(\alpha)+2 m^{2} \alpha^{2} \beta \lambda^{3} I_{1}(\alpha)-2 m^{4} \alpha^{2} \beta \lambda^{3} I_{1}(\alpha)-2 m^{2} \alpha^{3} \lambda^{4} I_{1}(\alpha)$ $+2 m^{4} \alpha^{3} \lambda^{4} I_{1}(\alpha)+8 I_{2}(\alpha)-24 m^{4} I_{2}(\alpha)+9 \alpha \beta \lambda I_{2}(\alpha)+8 m^{2} \alpha \beta \lambda I_{2}(\alpha)-m^{4} \alpha \beta \lambda I_{2}(\alpha)$ $+8 \lambda^{2} I_{2}(\alpha)+24 m^{4} \lambda^{2} I_{2}(\alpha)-\alpha^{2} \lambda^{2} I_{2}(\alpha)+m^{4} \alpha^{2} \lambda^{2} I_{2}(\alpha)-4 m^{2} \alpha \beta \lambda^{3} I_{2}(\alpha)+4 m^{4} \alpha \beta \lambda^{3} I_{2}(\alpha)$ $+2 m^{2} \alpha^{3} \beta \lambda^{3} I_{2}(\alpha)-2 m^{4} \alpha^{3} \beta \lambda^{3} I_{2}(\alpha)+4 m^{2} \alpha^{2} \lambda^{4} I_{2}(\alpha)-4 m^{4} \alpha^{2} \lambda^{4} I_{2}(\alpha)+\alpha^{2} \beta \lambda^{3} I_{1}(\alpha) \ln m$ $+3 m^{4} \alpha^{2} \beta \lambda^{3} I_{1}(\alpha) \ln m-\alpha^{3} \lambda^{4} I_{1}(\alpha) \ln m-3 m^{4} \alpha^{3} \lambda^{4} I_{1}(\alpha) \ln m-2 \alpha \beta \lambda I_{2}(\alpha) \ln m+6 m^{4} \alpha \beta \lambda I_{2}(\alpha) \ln m$ $+2 \alpha^{2} \lambda^{2} I_{2}(\alpha) \ln m-6 m^{4} \alpha^{2} \lambda^{2} I_{2}(\alpha) \ln m-2 \alpha \beta \lambda^{3} I_{2}(\alpha) \ln m-6 m^{4} \alpha \beta \lambda^{3} I_{2}(\alpha) \ln m+\alpha^{3} \beta \lambda^{3} I_{2}(\alpha) \ln m$ $+3 m^{4} \alpha^{3} \beta \lambda^{3} I_{2}(\alpha) \ln m+2 \alpha^{2} \lambda^{4} I_{2}(\alpha) \ln m+6 m^{4} \alpha^{2} \lambda^{4} I_{2}(\alpha) \ln m$.

For Cunningham/Mehta-Morse's model:

$$
\begin{aligned}
L_{11} & =\Delta_{M M} / \alpha \lambda\left(-\alpha \beta \lambda^{2} I_{1}(\alpha)+m^{4} \alpha \beta \lambda^{2} I_{1}(\alpha)+\alpha^{2} \lambda^{3} I_{1}(\alpha)-m^{4} \alpha^{2} \lambda^{3} I_{1}(\alpha)+2 \beta I_{2}(\alpha)\right. \\
& +2 m^{4} \beta I_{2}(\alpha)-2 \alpha \lambda I_{2}(\alpha)-2 m^{4} \alpha \lambda I_{2}(\alpha)+2 \beta \lambda^{2} I_{2}(\alpha)-2 m^{4} \beta \lambda^{2} I_{2}(\alpha)-\alpha^{2} \beta \lambda^{2} I_{2}(\alpha) \\
& \left.+m^{4} \alpha^{2} \beta \lambda^{2} I_{2}(\alpha)-2 \alpha \lambda^{3} I_{2}(\alpha)+2 m^{4} \alpha \lambda^{3} I_{2}(\alpha)\right) /(4 \gamma),
\end{aligned}
$$

where 
$\Delta_{M M}=4 \alpha \lambda^{2} I_{1}(\alpha)-4 m^{4} \alpha \lambda^{2} I_{1}(\alpha)-\alpha^{2} \beta \lambda^{3} I_{1}(\alpha)+2 m^{2} \alpha^{2} \beta \lambda^{3} I_{1}(\alpha)-m^{4} \alpha^{2} \beta \lambda^{3} I_{1}(\alpha)$

$+\alpha^{3} \lambda^{4} I_{1}(\alpha)-2 m^{2} \alpha^{3} \lambda^{4} I_{1}(\alpha)+m^{4} \alpha^{3} \lambda^{4} I_{1}(\alpha)-8 I_{2}(\alpha)-8 m^{4} I_{2}(\alpha)-7 \alpha \beta \lambda I_{2}(\alpha)+8 m^{2} \alpha \beta \lambda I_{2}(\alpha)$

$-m^{4} \alpha \beta \lambda I_{2}(\alpha)-8 \lambda^{2} I_{2}(\alpha)+8 m^{4} \lambda^{2} I_{2}(\alpha)-\alpha^{2} \lambda^{2} I_{2}(\alpha)+m^{4} \alpha^{2} \lambda^{2} I_{2}(\alpha)+2 \alpha \beta \lambda^{3} I_{2}(\alpha)-4 m^{2} \alpha \beta \lambda^{3} I_{2}(\alpha)$

$+2 m^{4} \alpha \beta \lambda^{3} I_{2}(\alpha)-\alpha^{3} \beta \lambda^{3} I_{2}(\alpha)+2 m^{2} \alpha^{3} \beta \lambda^{3} I_{2}(\alpha)-m^{4} \alpha^{3} \beta \lambda^{3} I_{2}(\alpha)-2 \alpha^{2} \lambda^{4} I_{2}(\alpha)+4 m^{2} \alpha^{2} \lambda^{4} I_{2}(\alpha)$

$-2 m^{4} \alpha^{2} \lambda^{4} I_{2}(\alpha)-\alpha^{2} \beta \lambda^{3} I_{1}(\alpha) \ln m+m^{4} \alpha^{2} \beta \lambda^{3} I_{1}(\alpha) \ln m+\alpha^{3} \lambda^{4} I_{1}(\alpha) \ln m-m^{4} \alpha^{3} \lambda^{4} I_{1}(\alpha) \ln m$

$+2 \alpha \beta \lambda I_{2}(\alpha) \ln m+2 m^{4} \alpha \beta \lambda I_{2}(\alpha) \ln m-2 \alpha^{2} \lambda^{2} I_{2}(\alpha) \ln m-2 m^{4} \alpha^{2} \lambda^{2} I_{2}(\alpha) \ln m+2 \alpha \beta \lambda^{3} I_{2}(\alpha) \ln m$

$-2 m^{4} \alpha \beta \lambda^{3} I_{2}(\alpha) \ln m-\alpha^{3} \beta \lambda^{3} I_{2}(\alpha) \ln m+m^{4} \alpha^{3} \beta \lambda^{3} I_{2}(\alpha) \ln m-2 \alpha^{2} \lambda^{4} I_{2}(\alpha) \ln m+2 m^{4} \alpha^{2} \lambda^{4} I_{2}(\alpha) \ln m$.

3.3.2. Hydrodynamic permeability of a porous cylinder-in-cell without impermeable core and with continuity condition

If $l=0$ and $\beta=0$, the cylindrical shell will reduce to a porous circular cylinder of radius $r=1(\tilde{r}=\tilde{b})$, with continuity conditions at the fluid-porous interface. In this case, taking into account different notations i.e., $\alpha=s, \lambda^{2}=m$ and substituting $\gamma^{2}$ instead of $\gamma$, we get the ear;ier deduced results [50] for hydrodynamic permeability $L_{11}$.

Happel's model

$L_{11}=\left[\lambda^{2} \alpha\left\{8\left(1+\gamma^{2}\right)+\lambda^{2} \alpha^{2}\left(\gamma^{2}-1\right)-\lambda^{2} \alpha^{2}\left(1+\gamma^{2}\right) \ln (\gamma)\right\} I_{1}(\alpha)+\right.$

$\left.2\left\{8+\lambda^{2}\left(\lambda^{2} \alpha^{2}-8\right)-\gamma^{2}\left(8+\lambda^{2}\left(8+\lambda^{2} \alpha^{2}\right)\right)+\lambda^{2} \alpha^{2}\left(-1+\lambda^{2}+\gamma^{2}\left(1+\lambda^{2}\right)\right) \ln (\gamma)\right\} \mathrm{I}_{2}(\alpha)\right] /$

$\left[8 \lambda^{4} \gamma \alpha^{3}\left(1+\gamma^{2}\right) \mathrm{I}_{1}(\alpha)-16 \lambda^{2} \gamma \alpha^{2}\left(-1+\lambda^{2}+\gamma^{2}\left(1+\lambda^{2}\right)\right) \mathrm{I}_{2}(\alpha)\right]$.

At $\lambda=1$ (equal viscosities) from $\mathrm{Eq}$ (68) we get the following expression for the hydrodynamic permeability:

$$
L_{11}=1 /\left\{\frac{8 \gamma \sigma^{2}\left[\sigma I_{1}(\sigma)\left(1+\gamma^{2}\right)-4 \gamma^{2} I_{2}(\sigma)\right]}{\sigma^{2}\left(\gamma^{2}-1\right)\left[\sigma I_{1}(\sigma)-2 I_{2}(\sigma)\right]+\left(8-\sigma^{2} \ln \gamma\right)\left[\sigma\left(1+\gamma^{2}\right) I_{1}(\sigma)-4 \gamma^{2} I_{2}(\sigma)\right]}\right\},
$$

which can be also obtained from earlier published paper by Deo and Yadav [49].

Kuwabara's model

$$
\begin{aligned}
& L_{11}=\left[2 \alpha\left\{16+\lambda^{2}\left(-16+\alpha^{2}(\gamma-1)\left(1+\gamma+\lambda^{2}(\gamma-3)\right)\right)+2 \lambda^{2} \alpha^{2}\left(\lambda^{2}-1\right) \ln (\gamma)\right\} \mathrm{I}_{0}(\alpha)+\right. \\
& \left\{-64+64 \lambda^{2}-4 \lambda^{2} \alpha^{2}\left(\gamma^{2}-5\right)-\lambda^{4} \alpha^{2}\left(3-4 \gamma+\gamma^{2}\right)\left(4+\alpha^{2}\right)-\right. \\
& \left.\left.2 \lambda^{2} \alpha^{2}\left(-4+\lambda^{2}\left(4+\alpha^{2}\right)\right) \ln (\gamma)\right\} \mathrm{I}_{1}(\alpha)\right] /\left[16 \gamma \lambda^{4} \alpha^{4} \mathrm{I}_{1}(\alpha)-32 \lambda^{2} \gamma \alpha^{3}\left(\lambda^{2}-1\right) \mathrm{I}_{2}(\alpha)\right] .
\end{aligned}
$$

For $\lambda=1$ we get the following expression for permeability from Eq (70):

$L_{11}=1 /\left\{\frac{16 \gamma \sigma^{2} I_{1}(\sigma)}{4 \sigma(\gamma-1)^{2} I_{0}(\sigma)-\left[\sigma^{2}\left(3-4 \gamma+\gamma^{2}+2 \ln \gamma\right)+8\left(\gamma^{2}-2 \gamma-1\right)\right] I_{1}(\sigma)}\right\}$,

which can also be also obtained using results from earlier published paper by Deo [46].

$\underline{\text { Kvashnin's model }}$ 
$L_{11}=\left[\left\{24-8 \gamma^{2}-8 \lambda^{2}\left(3+\gamma^{2}\right)+\lambda^{2} \alpha^{2}(\gamma-1)\left(1+\gamma-4 \lambda^{2}\right)+\lambda^{2} \alpha^{2}\left(-3+\gamma^{2}+\right.\right.\right.$

$\left.\left.\lambda^{2}\left(3+\gamma^{2}\right)\right) \ln (\gamma)\right\} \alpha \mathrm{I}_{0}(\alpha)+\left\{-48+16 \gamma^{2}+16 \lambda^{2}\left(3+\gamma^{2}\right)+2 \lambda^{2} \alpha^{2}\left(7+\gamma^{2}+4 \lambda^{2}(\gamma-1)\right)+\right.$

$\left.\left.2 \lambda^{4} \alpha^{4}(\gamma-1)-\lambda^{2} \alpha^{2}\left(-6+2 \gamma^{2}+2 \lambda^{2}\left(3+\gamma^{2}\right)+\lambda^{2} \alpha^{2}\left(3+\gamma^{2}\right) / 2\right) \ln (\gamma)\right\} I_{1}(\alpha)\right] /$

$\left[4 \gamma \lambda^{4} \alpha^{4}\left(3+\gamma^{2}\right) I_{1}(\alpha)-8 \gamma \lambda^{2} \alpha^{3}\left\{-3+\gamma^{2}+\lambda^{2}\left(3+\gamma^{2}\right)\right\} I_{2}(\alpha)\right]$.

At $\lambda=1$ (equal viscosities) from Eq (72) we get the following expression for permeability:

$L_{11}=\left\{2\left[\alpha^{3}(\gamma-3)(\gamma-1)-16 \gamma^{2} \alpha+2 \gamma^{2} \alpha^{3} \ln (\gamma)\right] \mathrm{I}_{0}(\alpha)+\left[4 \alpha^{4}(\gamma-1)+4 \alpha^{2}(\gamma+1)(\gamma+3)+\right.\right.$

$\left.\left.64 \gamma^{2}-\left(\gamma^{2} \alpha^{4}+3 \alpha^{4}+8 \gamma^{2} \alpha^{2}\right) \ln (\gamma)\right] \mathrm{I}_{1}(\alpha)\right\} /\left\{8 \alpha^{3}\left[\gamma \alpha\left(\gamma^{2}+3\right) \mathrm{I}_{1}(\alpha)-4 \gamma^{3} \mathrm{I}_{2}(\alpha)\right]\right\}$.

Cunnungham/Mehta-Morse's model

$$
\begin{aligned}
& L_{11}=\left[\left\{8\left(\lambda^{2}-1\right)-8 \gamma^{2}\left(1+\lambda^{2}\right)-\lambda^{2} \alpha^{2}(\gamma-1)\left(1+\gamma+2 \lambda^{2}(\gamma-1)\right)+\lambda^{2} \alpha^{2}\left(1+\gamma^{2}+\right.\right.\right. \\
& \left.\left.\lambda^{2}\left(\gamma^{2}-1\right)\right) \ln (\gamma)\right\} \alpha \mathrm{I}_{0}(\alpha)+\left\{16\left(1-\lambda^{2}+\gamma^{2}\left(1+\lambda^{2}\right)\right)+2 \lambda^{2} \alpha^{2}(\gamma-1)\left(3-2 \lambda^{2}+\right.\right. \\
& \left.\left.\left.\gamma\left(3+2 \lambda^{2}\right)\right)+\lambda^{4} \alpha^{4}(\gamma-1)^{2}-\lambda^{2} \alpha^{2}\left(4-\lambda^{2}\left(4+\alpha^{2}\right)+4 \gamma^{2}+\lambda^{2} \gamma^{2}\left(4+\alpha^{2}\right)\right) \ln (\gamma) / 2\right\} \mathrm{I}_{1}(\alpha)\right] / \\
& {\left[4 \gamma \lambda^{4} \alpha^{4}\left(\gamma^{2}-1\right) \mathrm{I}_{1}(\alpha)-8 \gamma \lambda^{2} \alpha^{3}\left(1-\lambda^{2}+\gamma^{2}\left(1+\lambda^{2}\right)\right) \mathrm{I}_{2}(\alpha)\right] .}
\end{aligned}
$$

At $\lambda=1$ from Eq (74) we get the following expression for the hydrodynamic permeability:

$L_{11}=\left\{\left[-2 \alpha^{3}(\gamma-1)(3 \gamma-1)-32 \gamma^{2} \alpha+4 \alpha^{3} \gamma^{2} \ln (\gamma)\right] \mathrm{I}_{0}(\alpha)+\left[2 \alpha^{4}(\gamma-1)^{2}+\right.\right.$

$\left.\left.4 \alpha^{2}(\gamma-1)(5 \gamma+1)+64 \gamma^{2}+\alpha^{2}\left(\alpha^{2}-\alpha^{2} \gamma^{2}-8 \gamma^{2}\right) \ln (\gamma)\right] \mathrm{I}_{1}(\alpha)\right\} /$

$\left\{8 \alpha^{3}\left[\alpha \gamma\left(\gamma^{2}-1\right) \mathrm{I}_{1}(\alpha)-4 \gamma^{3} \mathrm{I}_{2}(\alpha)\right]\right\}$.

\subsubsection{Hydrodynamic permeability of a membrane built up by solid cylinders}

At $l=1(\lambda \rightarrow \infty$ or $\sigma \rightarrow \infty)$, we have a membrane built up by solid impermeable cylindrical particles/fibres. The hydrodynamic permeability in this case reduces to the following expressions:

Happel's model [1]:

$$
L_{11}=1 /\left\{\frac{8 \gamma}{-(\ln \gamma+1)+2 \gamma^{2} /\left(1+\gamma^{2}\right)}\right\} .
$$

Kuwabara's model [1]:

$$
L_{11}=1 /\left\{\frac{8 \gamma}{-\log \gamma-(3 / 2)+2 \gamma-(1 / 2) \gamma^{2}}\right\} \text {. }
$$

Kvashnin's model:

$$
L_{11}=1 /\left\{4 \gamma\left(\gamma^{2}+3\right) /\left(2(\gamma-1)+\log \gamma\left(3-\gamma^{2}\right)\right\}\right. \text {. }
$$

Cunningham/Mehta-Morse's model:

$$
L_{11}=1 /\{4 \gamma(1+\gamma) /(\gamma-1-\log \gamma(1+\gamma))\} \text {. }
$$

\subsection{Results and discussion}


In this section we discuss the variation of hydrodynamic permeability of a membrane with permeability parameter, ratio of viscosities, jump coefficient and particle volume fraction for all four cell models.

On analyzing the effect of dimensionless permeability we observe that the hydrodynamic permeability decreases with $\sigma$, i.e. for highly permeable porous shells of the particles the hydrodynamic permeability of the membrane is higher (Fig.3). The hydrodynamic permeability is highest for Happel's model and lowest for Cunningham/Mehta-Morse's model. This dependence of hydrodynamic permeability for a porous medium built up by cylindrical particles matches the earlier results for a porous medium built up by spherical particles [40].

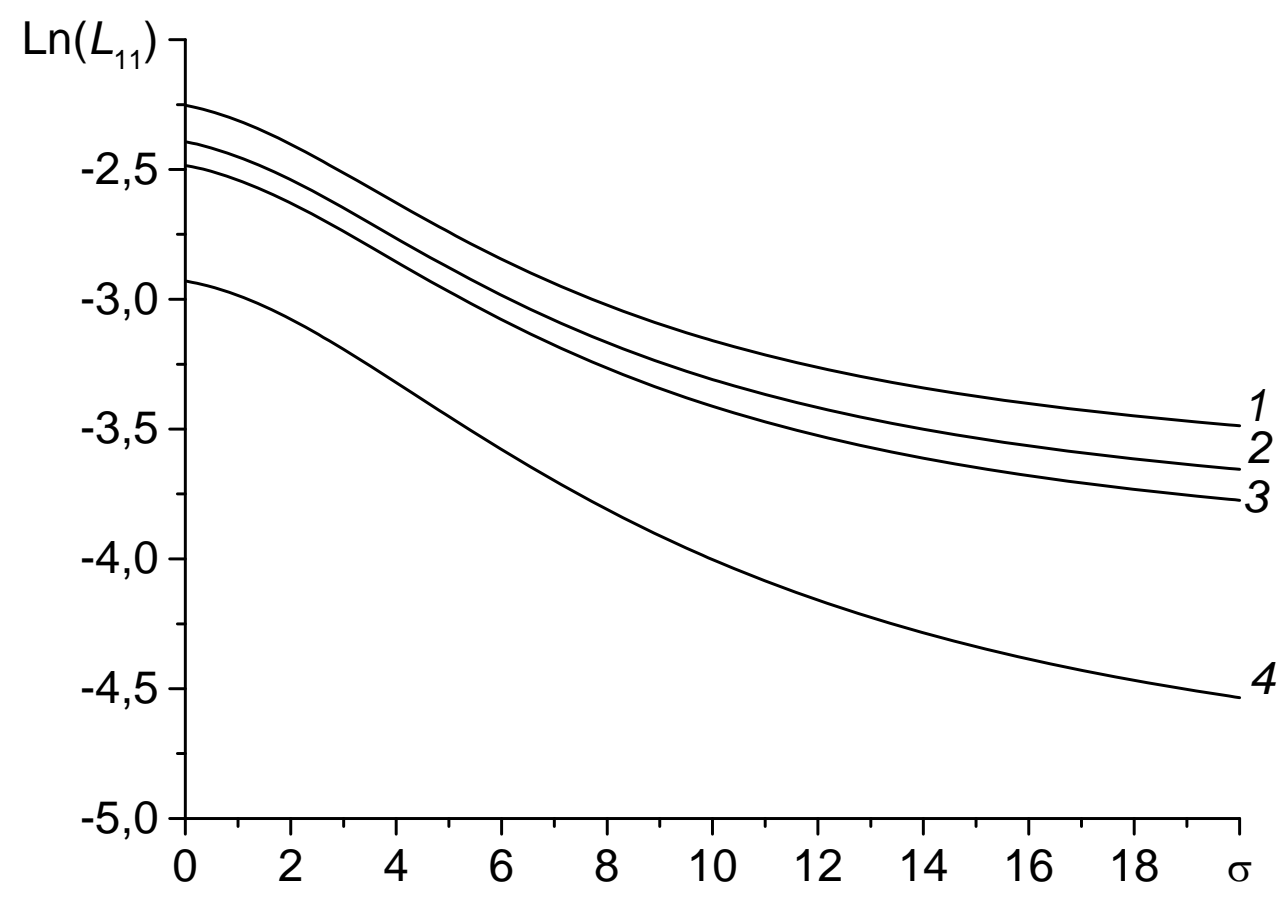

Fig. 3. Variation of dimensionless hydrodynamic permeability with $\sigma$ for $\gamma=0.5, \beta=-0.3, \lambda=2$ and $l=0.5$ for different models: 1 - Happel, 2 - Kvashnin, 3 Kuwabara, 4 - Cunningham/Mehta-Morse. 


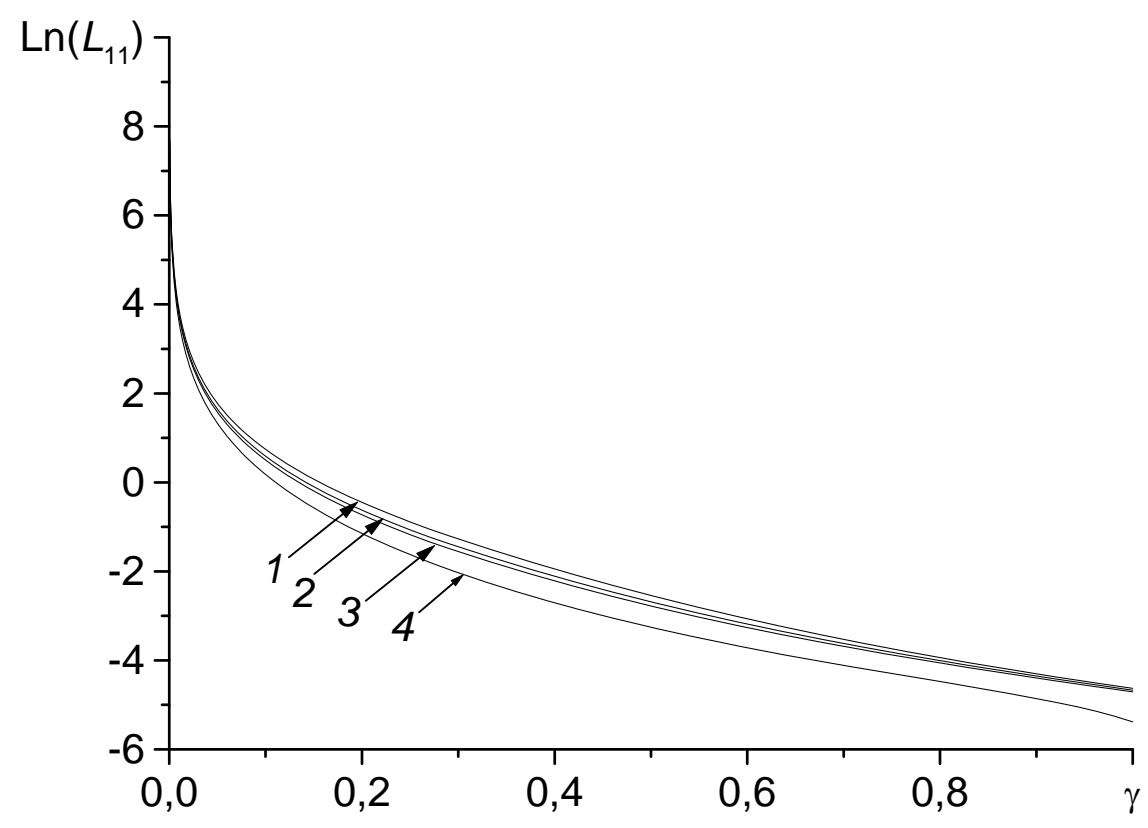

Fig. 4. Variation of dimensionless hydrodynamic permeability with the cylindrical particles volume fraction $\gamma$ for $\sigma=5, \beta=0.3, \lambda=2$ and $\ell=0.5$ for different models: 1 - Happel, 2 - Kvashnin, 3 Kuwabara, 4 - Cunningham/Mehta-Morse.

Fig. 4 shows that the hydrodynamic permeability of membrane decreases with particle volume fraction $\gamma$ and at low particle volume fraction, all four cell models agree. At $\gamma \rightarrow 0, L_{11}$ increases unboundedly and for $\gamma \rightarrow 1$ the membrane hydrodynamic permeability approaches zero, which is similar to the case of particles of spherical geometry [40]. Like in the case of spherical particles the hydrodynamic permeability is slightly lower for the Cunningham/Mehta-Morse's model. Other three models show a similar variation with particle volume fraction (Figs 3 and 4).

The dependence of the hydrodynamic permeability of membrane on parameter $\beta$ is presented in Fig. 5, which shows that $L_{11}$ increases as the jump coefficient $\beta$ increases. In other words, difference in shear stress at the fluid-porous layer interface has direct impact on the hydrodynamic permeability of the membrane and hence cannot be ignored. Moreover, behavior of the hydrodynamic permeability is not symmetric in relation to the sign of the jump coefficient $\beta$ as it could be expected from Eq (42). 


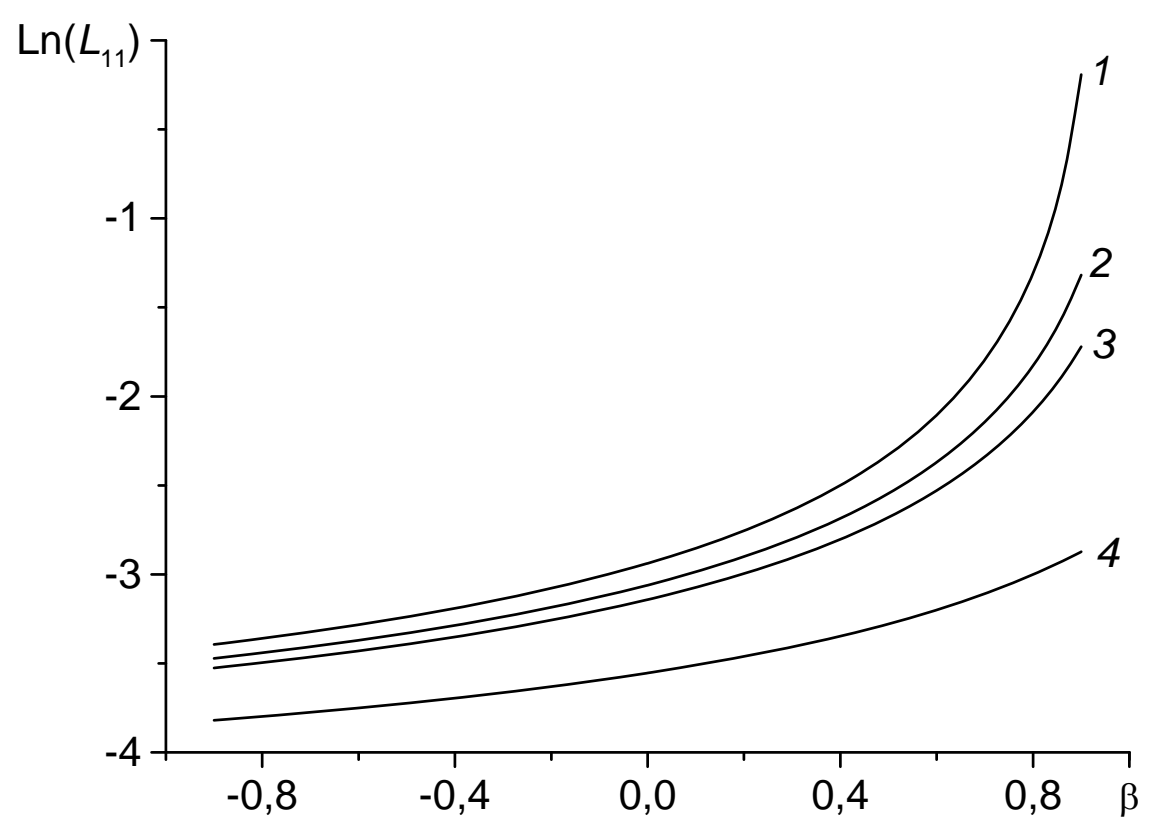

Fig. 5. Variation of dimensionless hydrodynamic permeability with $\beta$ for $\sigma=5, \gamma=0.64, \lambda=1$ and $l=0.5$ for different models: 1 - Happel, 2 - Kvashnin, 3 - Kuwabara, 4 - Cunningham/MehtaMorse

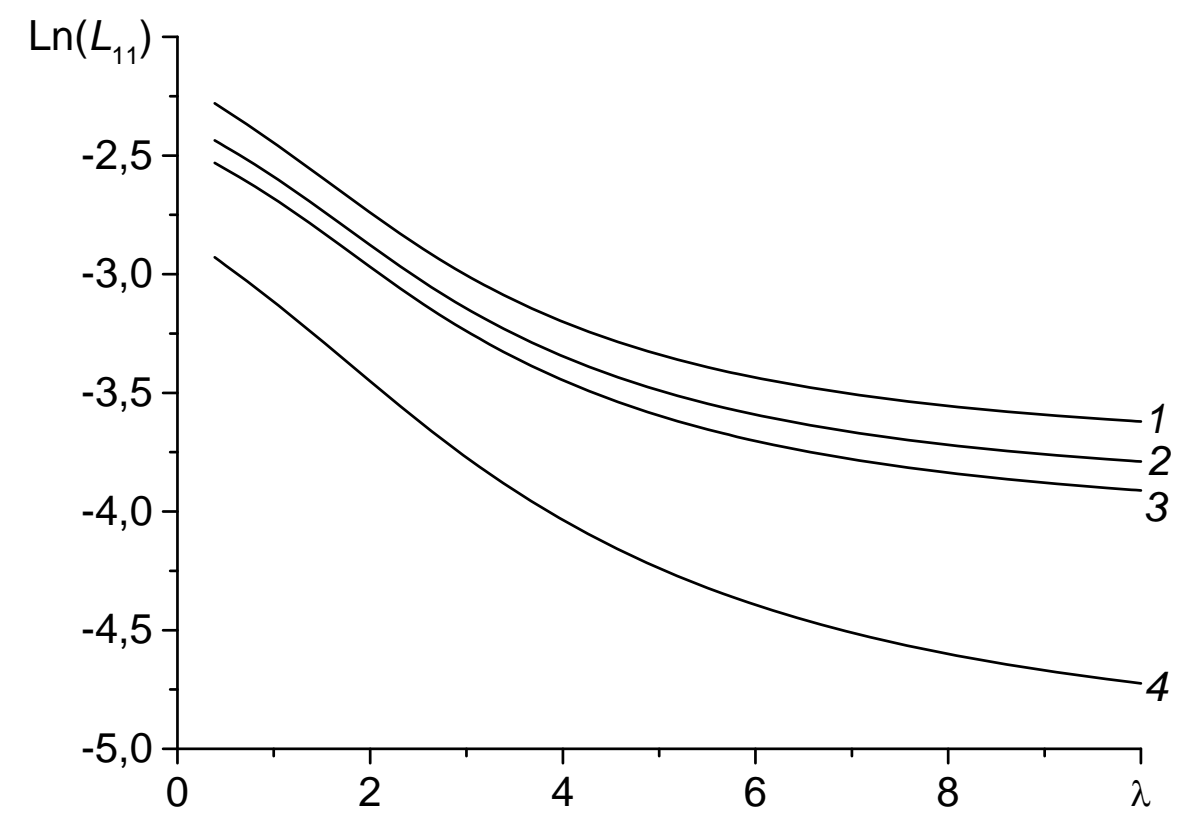

Fig. 6. Variation of dimensionless hydrodynamic permeability with $\lambda$ for $\sigma=5, \gamma=0.5, \beta=-0.3$ and $l=0.5$ for different models: 1 - Happel, 2 - Kvashnin, 3 Kuwabara, 4 - Cunningham/Mehta-Morse 
The influence of viscosity ratio on the hydrodynamic permeability of a membrane is shown in Fig. 6. The hydrodynamic permeability of a membrane decreases with the increase in the viscosity ratio $\lambda$, i.e. the hydrodynamic permeability of a membrane decreases with the increase in effective viscosity of the porous layers and with the decrease in viscosity of the clear fluid.

\section{Flow along parallel cylinders covered with a porous layer}

In this section, we consider the filtration through a membrane composed of cylinders covered with a porous layer when the flow is directed along the axis of cylinders (Fig. 7). As before, we consider the flow in an individual cell. Using a cylindrical system of coordinates where the axis is directed along the flow, equations that describe the flow outside and inside the porous layer have the following form:

$$
\begin{aligned}
& \tilde{\mu}^{(1)} \frac{1}{\tilde{r}} \frac{d}{d \tilde{r}}\left(\tilde{r} \frac{d \tilde{u}^{(1)}}{d \tilde{r}}\right)=\frac{d \tilde{p}}{d \tilde{z}}, \quad(\tilde{a}<\tilde{r}<\tilde{b}) \\
& \tilde{\mu}^{(2)} \frac{1}{\tilde{r}} \frac{d}{d \tilde{r}}\left(\tilde{r} \frac{d \tilde{u}^{(2)}}{d \tilde{r}}\right)-\frac{\tilde{\mu}^{(1)}}{\tilde{k}} \tilde{u}^{(2)}=\frac{d \tilde{p}}{d \tilde{z}}, \quad(\tilde{b}<\tilde{r}<\tilde{c})
\end{aligned}
$$

where $\tilde{u}^{(1)}, \tilde{u}^{(2)}$ are velocities of liquid in corresponding regions along the cylinder and $\frac{d \tilde{p}}{d \tilde{z}}$ is the pressure drop, which is considered to be constant and fixed by outer conditions.

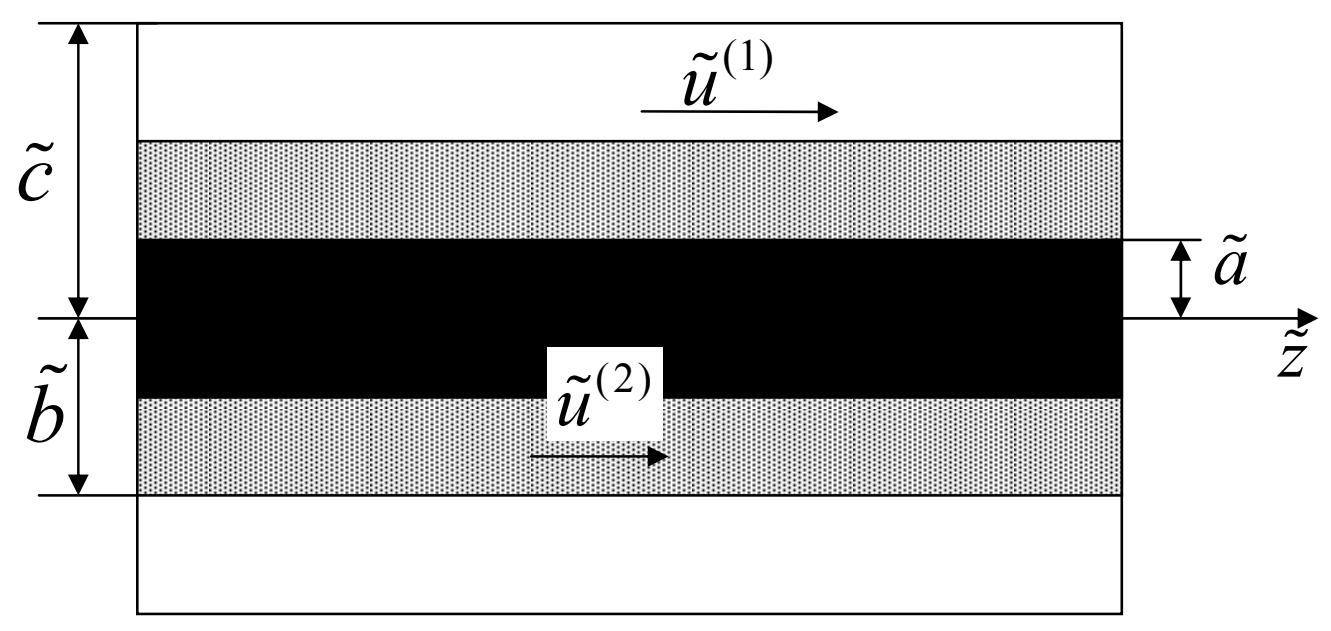

Fig.7. Schematic presentation of cylindrical cell with particle covered with porous layer at the imposed longitudinal flow of liquid.

We set the no-slip condition on the surface of a rigid cylinder as before

$$
\tilde{u}^{(2)}=0, \tilde{r}=\tilde{a} \text {. }
$$

At the liquid-porous layer interface, $\tilde{r}=\tilde{b}$, we use the continuity condition for velocity and the jump condition for the tangential stresses:

$$
\tilde{u}^{(1)}=\tilde{u}^{(2)},
$$




$$
\tilde{T}_{r z}^{(2)}-\tilde{T}_{r z}^{(1)}=\frac{\beta \tilde{\mu}^{(1)}}{\sqrt{\tilde{k}}} \tilde{u}^{(2)} .
$$

In this case, the Happel's, Kuwabara's, Kvashnin's, and Cunningham/Mehta-Morse's boundary conditions lead to the following single result:

$$
\frac{d \tilde{u}^{(1)}}{d \tilde{r}}=0 \text { at } \tilde{r}=\tilde{c} .
$$

Eqs (80) and (81) with boundary conditions (82)-(85) in dimensionless variables (21) have the following form:

$$
\begin{aligned}
& \frac{d}{d r}\left(r \frac{d u^{(1)}}{d r}\right)=r \omega, \\
& r \frac{d^{2} u^{(2)}}{d r^{2}}+\frac{d u^{(2)}}{d r}-r \alpha^{2} u^{i}=r \omega / \lambda^{2}, \\
& u^{(2)}=0 \text { at } r=l, \\
& u^{(1)}=u^{(2)} \text { at } r=1, \\
& \lambda^{2} \frac{d u^{(2)}}{d r}-\frac{d u^{(1)}}{d r}=\beta \sigma u^{(1)} \text { at } r=1, \\
& \frac{d u^{(1)}}{d r}=0 \text { at } r=m,
\end{aligned}
$$

where $\omega=\frac{d p}{d z}$ is introduced. The general solutions of Eqs. (86) and (87) can be expressed in the following form:

$$
\begin{aligned}
& u^{(1)}=b_{1}+b_{2} \ln r+\omega \frac{r^{2}}{4}, \\
& u^{(2)}=c_{1} \mathrm{I}_{0}(s r)+c_{2} \mathrm{~K}_{0}(s r)-\frac{\omega}{\lambda^{2} \alpha^{2}} .
\end{aligned}
$$

Substituting expressions (92) and (93) into boundary conditions (88)-(91) we find the following values of constants $b_{1}, b_{2}, c_{1}$ and $c_{2}$ as

$$
\begin{aligned}
& b_{1}=-\omega\left\{\alpha ^ { 2 } \left[\left((2-\alpha \beta \gamma \lambda) \mathrm{I}_{0}(\alpha)+\gamma\left(\alpha \lambda^{2} \mathrm{I}_{1}(\alpha)-2 \mathrm{I}_{2}(\alpha)\right)\right) \mathrm{K}_{0}(\alpha l)+\right.\right. \\
&\left.\left.\mathrm{I}_{0}(\alpha l)\left((\alpha \beta \gamma \lambda-2) \mathrm{K}_{0}(\alpha)+\gamma\left(\alpha \lambda^{2} \mathrm{~K}_{1}(\alpha)+2 \mathrm{~K}_{2}(\alpha)\right)\right)\right]-4 \gamma\right\} / \\
&\left\{4 \alpha^{3} \gamma \lambda\left[\left(\lambda \mathrm{I}_{1}(\alpha)-\beta \mathrm{I}_{0}(\alpha)\right) \mathrm{K}_{0}(\alpha l)+\mathrm{I}_{0}(\alpha l)\left(\beta \mathrm{K}_{0}(\alpha)+\lambda \mathrm{K}_{1}(\alpha)\right)\right]\right\}, \\
& b_{2}=-\frac{\omega}{2 \gamma}, \\
& c_{1}=\frac{\omega\left\{2 \beta \gamma \mathrm{K}_{0}(\alpha)+[\alpha \lambda(\gamma-1)-2 \beta \gamma] \mathrm{K}_{0}(\alpha l)+2 \gamma \lambda \mathrm{K}_{1}(\alpha)\right\}}{2 \alpha^{2} \gamma \lambda^{2}\left\{\left[\lambda \mathrm{I}_{1}(\alpha)-\beta \mathrm{I}_{0}(\alpha)\right] \mathrm{K}_{0}(\alpha l)+\left[\lambda \mathrm{K}_{1}(\alpha)+\beta \mathrm{K}_{0}(\alpha)\right] \mathrm{I}_{0}(\alpha l)\right\}} \\
& c_{2}=\frac{\omega\left\{2 \gamma \lambda \mathrm{I}_{1}(\alpha)-2 \beta \gamma \mathrm{I}_{0}(\alpha)+[2 \beta \gamma-\alpha \lambda(\gamma-1)] \mathrm{I}_{0}(\alpha l)\right\}}{2 \alpha^{2} \gamma \lambda^{2}\left\{\left[\lambda \mathrm{I}_{1}(\alpha)-\beta \mathrm{I}_{0}(\alpha)\right] \mathrm{K}_{0}(\alpha l)+\left[\lambda \mathrm{K}_{1}(\alpha)+\beta \mathrm{K}_{0}(\alpha)\right] \mathrm{I}_{0}(\alpha l)\right\}}
\end{aligned}
$$

The dimensionless flow rate of fluid passing through the cylindrical cell is equal to (see Fig. 7): 


$$
Q=2 \pi\left(\int_{l}^{1} u^{(2)} r d r+\int_{1}^{m} u^{(1)} r d r\right)
$$

In the case under consideration, the filtration rate is equal to $V_{f}=Q /(\pi / \gamma)$ [1]. Using the Darcy's law for the flow in porous medium, we obtain in dimensionless form

$$
V_{f}=-L_{11} d p / d x=-L_{11} \omega
$$

From expressions (92)-(99), we derive the following formula for the coefficient of hydrodynamic permeability $L_{11}$ of the porous medium under consideration:

$$
\begin{aligned}
& L_{11}=(-\gamma / \omega)\left\{\left[\omega\left(l^{2}-1\right)+2 \alpha \lambda^{2}\left(c_{1} \mathrm{I}_{1}(\alpha)-c_{1} l \mathrm{I}_{1}(\alpha l)-c_{2} \mathrm{~K}_{1}(\alpha)+\right.\right.\right. \\
& \left.\left.\left.c_{2} l \mathrm{~K}_{1}(\alpha l)\right)\right] /\left(\alpha^{2} \lambda^{2}\right)+\left[-4 b_{2} \gamma \ln (\gamma)-(\gamma-1)\left(\gamma\left(8 b_{1}-4 b_{2}+\omega\right)+\omega\right)\right] /\left(8 \gamma^{2}\right)\right\} .
\end{aligned}
$$

Let us consider now the most significant limiting cases of general equation (100).

\subsection{Set of porous particles}

At $l=0$, we have a porous particle with no rigid impermeable core and the expression for hydrodynamic permeability can be written as

$$
\begin{aligned}
& L_{11}=\left\{-8 \beta \gamma^{2} I_{2}(\alpha)-\lambda I_{1}(\alpha)\left[\alpha^{2} \lambda^{2}\left(\gamma^{2}-4 \gamma+3\right)+2 \alpha^{2} \lambda^{2} \ln (\gamma)+8 \gamma(\gamma-2)\right]+\right. \\
& \left.\alpha \lambda I_{0}(\alpha)\left[4(\gamma-1)^{2}+\alpha \beta \lambda\left(\gamma^{2}-4 \gamma+3\right)+2 \alpha \beta \lambda \ln (\gamma)\right]\right\} /\left\{8 \alpha^{2} \lambda^{2} \gamma\left[\lambda I_{1}(\alpha)-\beta I_{0}(\alpha)\right]\right\} .
\end{aligned}
$$

\subsection{Set of solid impermeable particles}

At $\sigma \rightarrow \infty$, or $\lambda \rightarrow \infty$, or $l=1$ we have a particle without a porous layer that is absolutely impermeable to liquid. In this case the expression for the hydrodynamic permeability acquires the known form [1]

$$
L_{11}=\frac{-2 \ln (\gamma)-\gamma^{2}+4 \gamma-3}{8 \gamma} \text {. }
$$




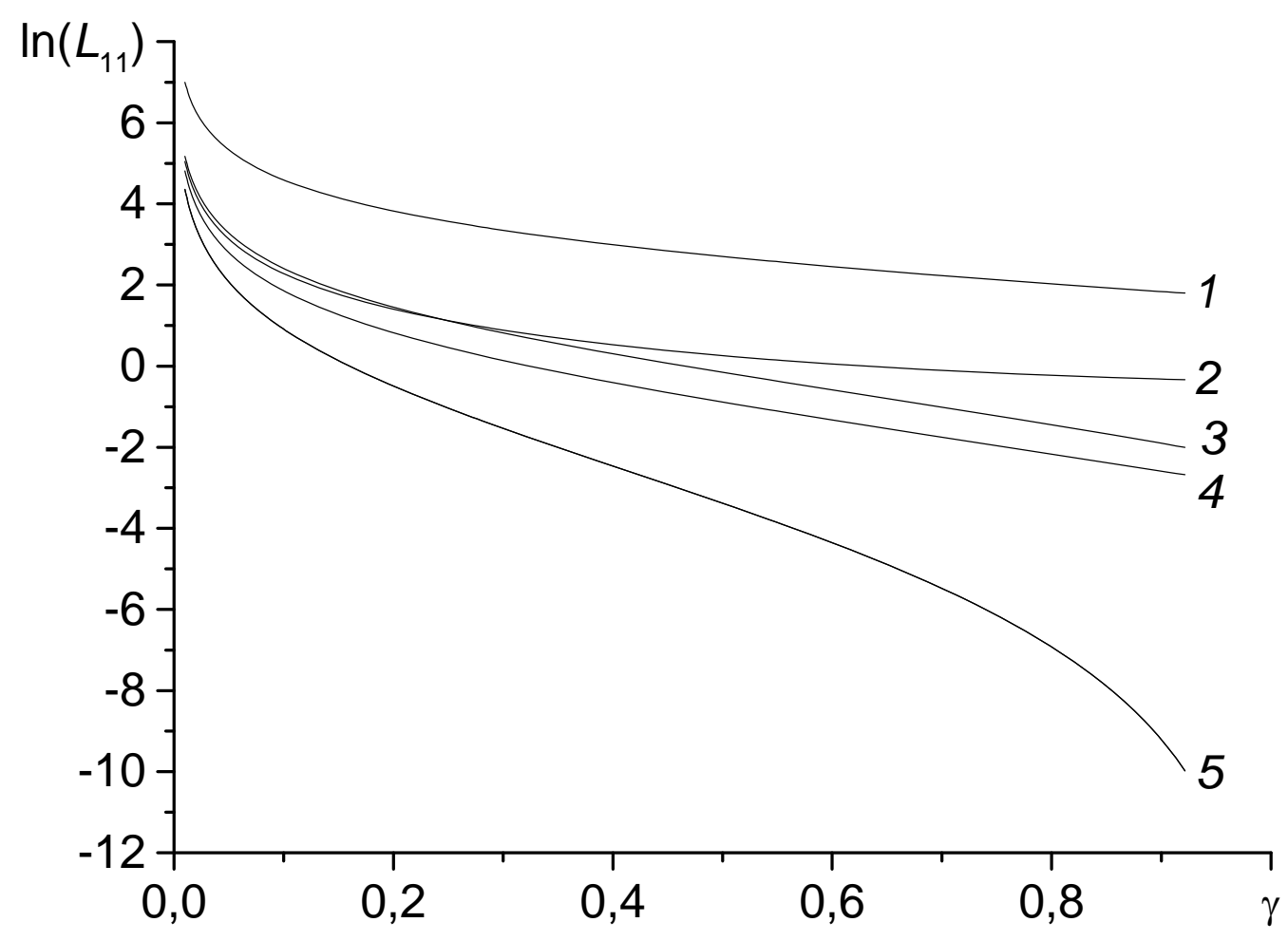

Fig. 8. Dependences of the dimensionless hydrodynamic permeability $L_{11}$ of a membrane composed by cylindrical particles on imposed longitudinal flow of liquid on the particle volume fraction $\gamma$ at $\lambda=0.5 ; \alpha=2: 1$ - completely porous particle at $\beta=0.3$ and $2-\beta=-0.3,3$ - partially porous particles $(l=0.7$ ) at $\beta=0.3$ and $4-\beta=-0.3$ (curve 4 ), 5 - solid impermeable particles.

Fig.8 demonstrates the dependences of the dimensionless hydrodynamic permeability $L_{11}$ of the membrane composed by cylindrical particles on the p[article volume fraction $\gamma$ at $\lambda=0.5 ; l=0.7 ; \alpha=2$ for different conditions. Curves $1-5$ are plotted using formulas (100)(102). The permeability of the membrane decreases with increasing $\gamma$, i.e., with the increase in the fraction of solid phase. The curves 3 and 4 for particles covered with a porous layer are located lower than curves 1 and 2 for completely porous particles and higher than curve 5 for impermeable solid particles. The latter is easy to understand: the larger the fraction of porous medium, the higher the permeability. As before, when $\gamma \rightarrow 0$, the permeability of the membrane increases infinitely for all models. When $\gamma \rightarrow 1$ for the model with rigid impermeable particles the permeability tends to zero (curve 5), which also follows from formula (102). When $\gamma \rightarrow 1$ the medium formed by completely porous particles (curves 1 and 2) is transformed into the Brinkman's medium and the permeability tends to its limiting value

$$
L_{11}=\frac{\lambda \mathrm{I}_{1}(\alpha)-\beta \mathrm{I}_{2}(\alpha)}{\alpha^{2} \lambda^{2}\left[\lambda \mathrm{I}_{1}(\alpha)-\beta \mathrm{I}_{0}(\alpha)\right]},
$$


which follows from formula (101).

In the case of the particle with porous layer (curves 3 and 4) at $\gamma \rightarrow 1$, we deal with the flow of fluid in the Brinkman's medium consisting of impermeable cylindrical fibers. According to the formula (100), the hydrodynamic permeability of such medium is equal to

$$
\begin{aligned}
& L_{11}=\left\{\left\{l^{2}\left[-\beta \mathrm{K}_{0}(\alpha)-\lambda \mathrm{K}_{1}(\alpha)\right] \mathrm{I}_{2}(\alpha l)+l^{2}\left[\beta \mathrm{I}_{0}(\alpha)-\lambda \mathrm{I}_{1}(\alpha)\right] \mathrm{K}_{2}(\alpha l)-\right.\right. \\
& \left.\left.\beta \mathrm{I}_{2}(\alpha) \mathrm{K}_{0}(\alpha l)+\lambda\left[\mathrm{I}_{1}(\alpha) \mathrm{K}_{0}(\alpha l)+\mathrm{I}_{0}(\alpha l) \mathrm{K}_{1}(\alpha)\right]+\beta \mathrm{I}_{0}(\alpha l) \mathrm{K}_{2}(\alpha)\right\} \alpha^{2}-4 \beta\right\} / \\
& \left\{\alpha^{4} \lambda^{2}\left\{\left[\lambda \mathrm{I}_{1}(\alpha)-\beta \mathrm{I}_{0}(\alpha)\right] \mathrm{K}_{0}(\alpha l)+\left[\beta \mathrm{K}_{0}(\alpha)+\lambda \mathrm{K}_{1}(\alpha)\right] \mathrm{I}_{0}(\alpha l)\right\}\right\} .
\end{aligned}
$$

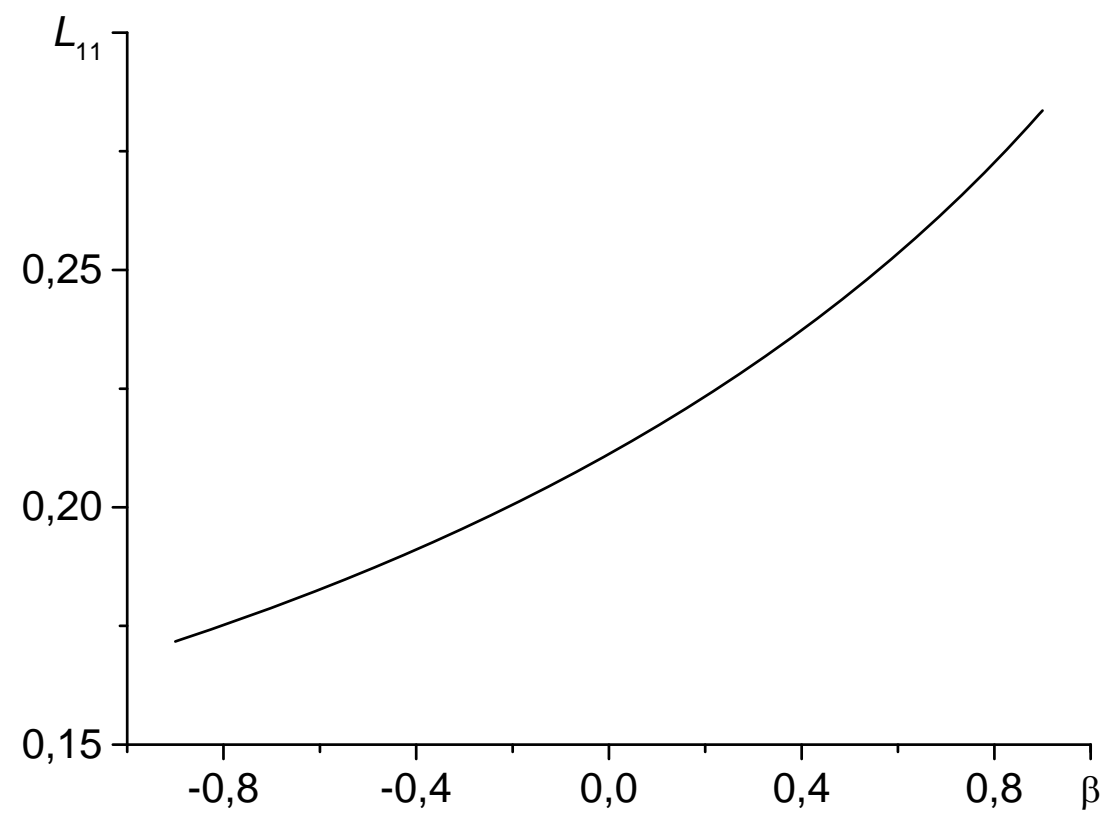

Fig. 9. Variation of dimensionless hydrodynamic permeability $L_{11}$ of a membrane consisting of cylindrical particles under longitudinal flow on jump coefficient $\beta$ for $\sigma=1, \quad \ell=0.5, \quad \lambda^{2}=2, \gamma=0.5$.

The hydrodynamic permeability increases with jump coefficient $\beta$ as shown in Fig.9, which shows lower resistance of the membrane against the imposed flow for a relatively higher shear stress in porous region compared to the clear fluid region. 


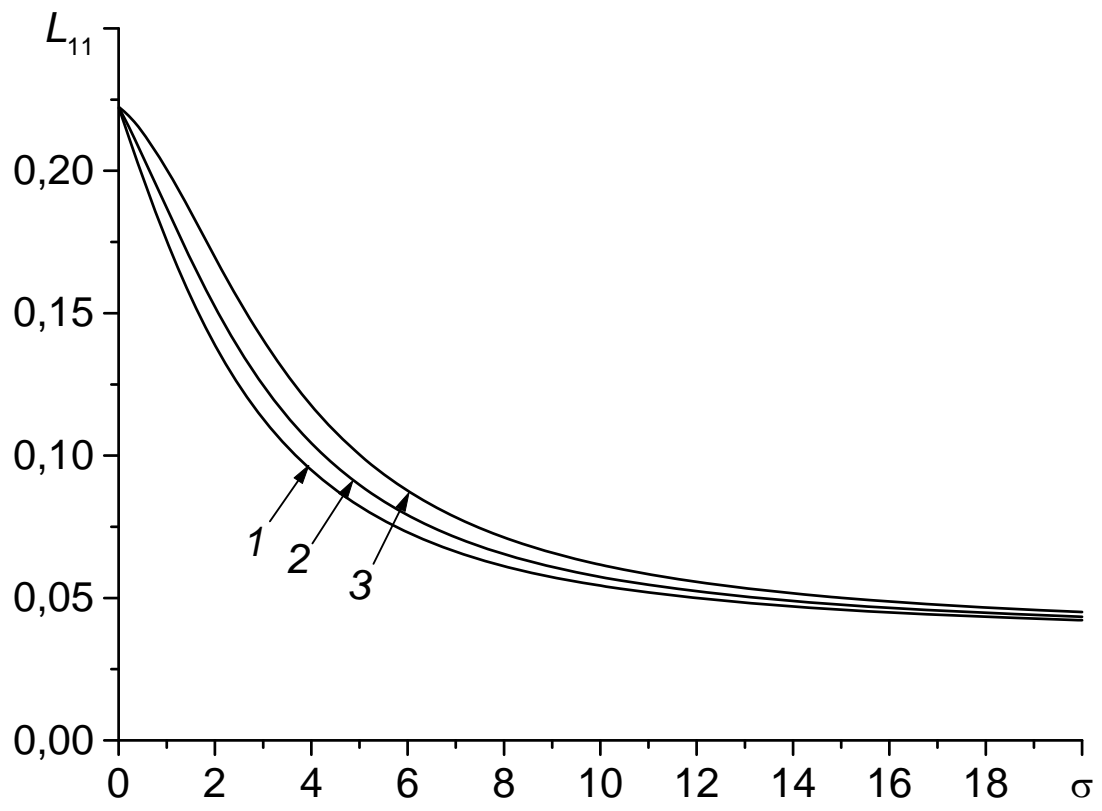

Fig. 10. Variation of dimensionless hydrodynamic permeability $L_{11}$ of a membrane built up by cylindrical particles under longitudinal flow on dimensionless $\sigma$ for $\ell=0.5, \lambda^{2}=2, \gamma=0.5$ and $\beta=-0.8(1),-0.5(2),-0.2(3)$

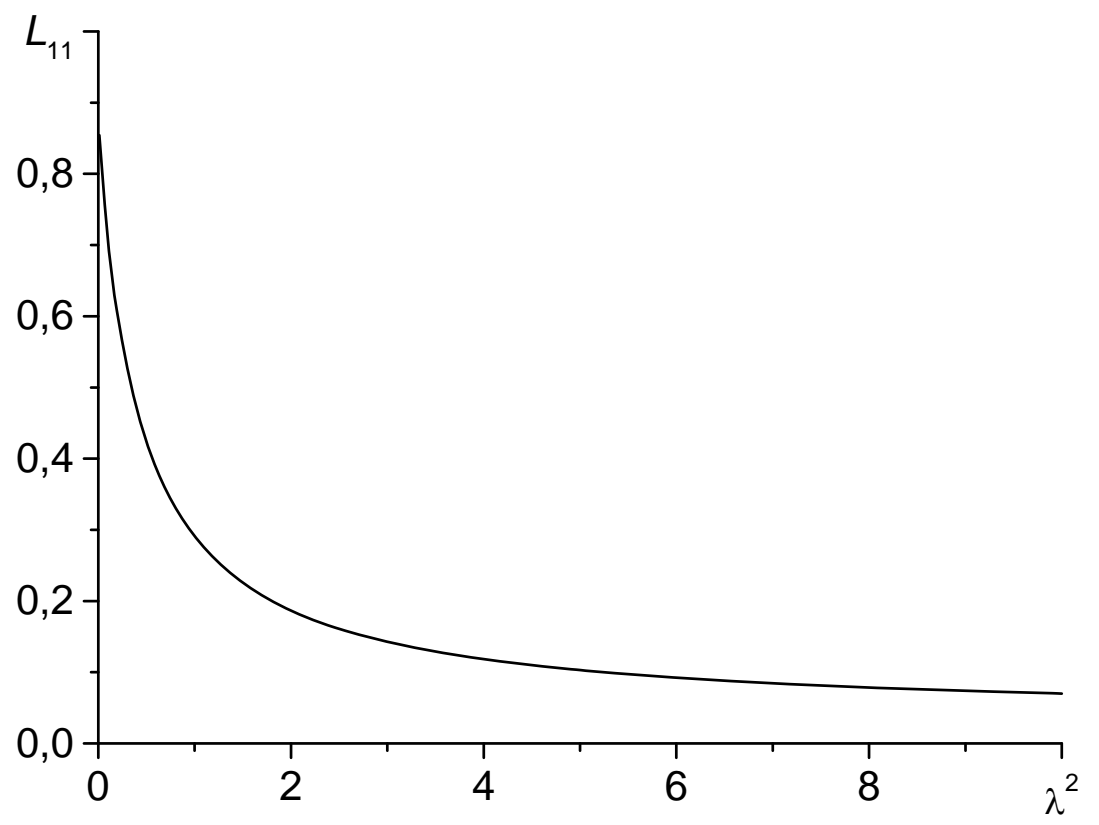

Fig. 11. Variation of dimensionless hydrodynamic permeability $L_{11}$ of a membrane consisting of cylindrical particles under longitudinal flow on viscosity ratio $\lambda^{2}$ for $\sigma=1, \ell=0.5, \quad \beta=-0.5, \gamma=0.5$ 
The variation of hydrodynamic permeability with dimensionless resistance of Brinkman medium, $\sigma$, is shown in Fig.10. The latter figure shows that with the increase in dimensionless resistance $\sigma$ the hydrodynamic permeability is decreasing. An almost similar observation for the drag force has been reported by Bhattacharya and Raja-Shekhar [53].

Fig.11 shows the decay in hydrodynamic permeability with viscosity ratio which agrees with previous results for spherical particles and cylindrical particles for flow across the membrane.

\section{Comparison of different cell models}

In this section, we compare different cell models that describe the flow in porous media, i.e., we consider the flows through the membranes formed by spherical and cylindrical particles with longitudinal, transverse, or random orientations toward the flow.

\subsection{Composite particles with a porous layer on the surface}

Table 1 lists the values of permeability $L_{11}$ for membranes composed of rigid spherical particles or cylinders covered with porous layers at different porosities $\varepsilon$ and fixed values of $\lambda=\sqrt{2}, l=0.4, \sigma=3, \beta=0$. The permeability of porous medium formed by spherical particles was calculated using equations reported in [40].

Table 1. Values of the dimensionless permeability $L_{11}$ for membranes composed of spherical particles or cylinders covered with a porous layer*

\begin{tabular}{|c|c|c|c|c|c|c|c|c|c|c|c|c|c|}
\hline Model & \multicolumn{9}{|c|}{ Spherical particles } & \multicolumn{10}{|c|}{ Cylinders } \\
\hline Orientation & \multicolumn{3}{|c|}{} & \multicolumn{1}{|c|}{ Normal } & \multicolumn{1}{c|}{ Parallel } & \multicolumn{4}{c|}{ Random } \\
\hline Bound. Cond. & $\mathrm{H}$ & $\mathrm{Kv}$ & $\mathrm{Ku}$ & $\mathrm{Cu}$ & $\mathrm{H}$ & $\mathrm{Kv}$ & $\mathrm{Ku}$ & $\mathrm{Cu}$ & & $\mathrm{H}$ & $\mathrm{Kv}$ & $\mathrm{Ku}$ & $\mathrm{Cu}$ \\
\hline Porosity & 1 & 2 & 3 & 4 & 5 & 6 & 7 & 8 & 9 & 10 & 11 & 12 & 13 \\
\hline 0.9 & 1.66 & 1.55 & 1.48 & 1.21 & 2.54 & 2.22 & 2.05 & 1.57 & 3.66 & 2.91 & 2.70 & 2.59 & 2.27 \\
\hline 0.8 & 0.65 & 0.60 & 0.57 & 0.45 & 0.85 & 0.73 & 0.67 & 0.48 & 1.14 & 0.94 & 0.86 & 0.82 & 0.70 \\
\hline 0.7 & 0.36 & 0.33 & 0.32 & 0.25 & 0.41 & 0.35 & 0.32 & 0.22 & 0.53 & 0.45 & 0.41 & 0.39 & 0.33 \\
\hline 0.6 & 0.23 & 0.21 & 0.20 & 0.16 & 0.23 & 0.20 & 0.19 & 0.13 & 0.29 & 0.25 & 0.23 & 0.22 & 0.18 \\
\hline 0.5 & 0.16 & 0.15 & 0.14 & 0.12 & 0.15 & 0.13 & 0.12 & 0.08 & 0.18 & 0.16 & 0.15 & 0.14 & 0.12 \\
\hline 0.4 & 0.12 & 0.11 & 0.11 & 0.09 & 0.10 & 0.09 & 0.08 & 0.06 & 0.12 & 0.11 & 0.10 & 0.09 & 0.08 \\
\hline 0.3 & 0.09 & 0.09 & 0.08 & 0.07 & 0.07 & 0.06 & 0.06 & 0.04 & 0.09 & 0.07 & 0.07 & 0.07 & 0.06 \\
\hline 0.2 & 0.07 & 0.07 & 0.07 & 0.06 & 0.05 & 0.05 & 0.04 & 0.03 & 0.07 & 0.06 & 0.05 & 0.05 & 0.04 \\
\hline 0.1 & 0.06 & 0.06 & 0.06 & 0.05 & 0.04 & 0.04 & 0.03 & 0.02 & 0.05 & 0.04 & 0.04 & 0.04 & 0.03 \\
\hline
\end{tabular}

*Abbreviations for models: H for Happel's, Kv for Kvashnin's, Ku for Kuwabara's and Cu for Cunningham/Mehta-Morse's models 
The permeability of a medium formed by cylinders was calculated using equations (59) and (100). For the random orientation of the cylinder, we used an averaging procedure based on the following considerations. When cylinders are oriented perpendicular to the flow, the model yields the same results, which were deduced for cylinders with longitudinal or random orientations in the plane normal to the flow. Therefore, when considering the case of random orientation, the averaging was performed so that the weight of the contribution from the cylinders with normal orientation to the flow was twice as large as that of the flow along the cylinders. Thus obtained values of permeability are listed in columns of Table 1 referred to the cases of random packing.

The following conclusions can be drawn from the values of permeability listed in Table 1 . At a small fraction of solid phase (upper lines in Table 1), the permeability of a porous medium formed by cylinders is higher than for media composed by spherical particles. Moreover, the system of cylinders oriented along the flow is characterized by the largest permeability. The values of permeability for different systems are leveled with an increase in the concentration of solid phase (with a decrease in porosity). This can be explained by the fact that geometric differences between media formed by spherical particles and cylinders become less noticeable at high concentrations of solid phase. At the concentration of solid phase equals $0.9(\varepsilon=0.1)$, the values of dimensionless permeability vary from 0.002 to 0.006 . Note also that, unlike weakly concentrated media, at high concentration of solid phase, the values of hydrodynamic permeability appeared to be larger for the set of spherical particles.

\subsection{Completely porous particles}

The values of the dimensionless permeability $L_{11}$ of membranes formed by porous spherical particles or cylinders at different values of porosity $\varepsilon$ and $\lambda=\sqrt{3}, \sigma=2$ are shown in Table 2 .

Table 2 is constructed analogously to Table 1 . The behavior of permeability $L_{11}$ for media formed by completely porous and composite particles and cylinders is very similar. Furthermore, the dependence on the type of boundary conditions becomes less marked and almost vanishes at high concentration of solid phase $(\varepsilon<0.4)$. The values of the permeability do not tend to zero with a similar tendency of porosity, since, in this case, the fluid can flow through porous shells.

Table 2. Values of the dimensionless permeability $L_{11}$ for membranes composed of porous spherical particles or cylinders 


\begin{tabular}{|c|c|c|c|c|c|c|c|c|c|c|c|c|c|}
\hline Model & \multicolumn{10}{|c|}{ Spherical particles } & \multicolumn{10}{|c|}{ Cylinders } \\
\hline Orientation & \multicolumn{3}{|c|}{} & \multicolumn{1}{|c|}{ Normal } & \multicolumn{1}{c|}{ Parallel } & \multicolumn{4}{c|}{ Random } \\
\hline Bound.cond. & $\mathrm{H}$ & $\mathrm{Kv}$ & $\mathrm{Ku}$ & $\mathrm{Cu}$ & $\mathrm{H}$ & $\mathrm{Kv}$ & $\mathrm{Ku}$ & $\mathrm{Cu}$ & & $\mathrm{H}$ & $\mathrm{Kv}$ & $\mathrm{Ku}$ & $\mathrm{Cu}$ \\
\hline Porosity & 1 & 2 & 3 & 4 & 5 & 6 & 7 & 8 & 9 & 10 & 11 & 12 & 13 \\
\hline 0.9 & 3.39 & 3.30 & 3.24 & 3.00 & 4.43 & 4.13 & 3.98 & 3.53 & 5.31 & 4.73 & 4.53 & 4.43 & 4.12 \\
\hline 0.8 & 1.52 & 1.49 & 1.47 & 1.37 & 1.80 & 1.70 & 1.65 & 1.49 & 1.99 & 1.87 & 1.80 & 1.76 & 1.65 \\
\hline 0.7 & 0.95 & 0.93 & 0.92 & 0.88 & 1.06 & 1.01 & 0.99 & 0.91 & 1.11 & 1.08 & 1.05 & 1.03 & 0.98 \\
\hline 0.6 & 0.68 & 0.67 & 0.67 & 0.64 & 0.73 & 0.70 & 0.69 & 0.66 & 0.75 & 0.73 & 0.72 & 0.71 & 0.69 \\
\hline 0.5 & 0.53 & 0.52 & 0.52 & 0.51 & 0.55 & 0.54 & 0.53 & 0.51 & 0.55 & 0.55 & 0.54 & 0.54 & 0.53 \\
\hline 0.4 & 0.43 & 0.43 & 0.43 & 0.42 & 0.44 & 0.43 & 0.43 & 0.42 & 0.44 & 0.44 & 0.44 & 0.43 & 0.43 \\
\hline 0.3 & 0.36 & 0.36 & 0.36 & 0.36 & 0.37 & 0.36 & 0.36 & 0.36 & 0.37 & 0.37 & 0.36 & 0.36 & 0.36 \\
\hline 0.2 & 0.31 & 0.31 & 0.31 & 0.31 & 0.32 & 0.31 & 0.31 & 0.31 & 0.32 & 0.32 & 0.31 & 0.31 & 0.31 \\
\hline 0.1 & 0.28 & 0.28 & 0.28 & 0.28 & 0.28 & 0.28 & 0.28 & 0.28 & 0.28 & 0.28 & 0.28 & 0.28 & 0.28 \\
\hline
\end{tabular}

\subsection{Rigid impermeable particles}

The semi-empirical Kozeny-Karman formula [1] results in the following expression for the permeability of porous media:

$$
L_{11}=\frac{\varepsilon \rho^{2}}{k \tilde{b}^{2}},
$$

where $k$ is the dimensionless Kozeny constant; $\rho$ is the hydraulic radius, which is equal to the ratio of pore volume to the wetting area, and $\varepsilon$ is the porosity.

The calculation of the theoretical values of the Kozeny constant using formula (105) yields the following relation:

$$
k=\frac{\varepsilon \rho^{2}}{\tilde{b}^{2} L_{11}} .
$$

For media composed of cylindrical particles, we have

$$
\rho=\frac{\pi\left(\tilde{c}^{2}-\tilde{b}^{2}\right)}{2 \pi \tilde{b}}=\frac{\tilde{b}}{2}\left(\frac{1-\gamma}{\gamma}\right)=\frac{\varepsilon \tilde{b}}{2(1-\varepsilon)} .
$$

Substituting expression (107) into formula (106), we obtain

$$
k=\frac{\varepsilon^{3}}{4(1-\varepsilon)^{2} L_{11}},
$$

where $L_{11}$ is found from one of formulas (76) - (79) or (102).

For media composed of spherical particles [40], we have

$$
\rho=\frac{\varepsilon \tilde{a}}{3(1-\varepsilon)}
$$


and the relation for the Kozeny constant can be expressed as

$$
k=\frac{\varepsilon^{3}}{9(1-\varepsilon)^{2} L_{11}},
$$

where $L_{11}$ is calculated using one of of formulas from [40].

Table 3 presents the theoretical values of the Kozeny constant $k$ for membranes formed by spherical particles or cylinders at different values of porosity $\varepsilon$. According to the current hypothesis, the Kozeny constant should be a constant independent of both the structure of membrane and its porosity.

Table 3. Theoretical values of the Kozeny constant $k$ for membranes composed of solid spherical particles or cylinders

\begin{tabular}{|c|c|c|c|c|c|c|c|c|c|c|c|c|c|}
\hline Model & & herical & particl & & & & & & Cylinders & & & & \\
\hline Orientation & & & & & & Nor & $\mathrm{mal}$ & & Parallel & & Cha & otic & \\
\hline Bound. Cond. & $\mathrm{H}$ & $\mathrm{Kv}$ & $\mathrm{Ku}$ & $\mathrm{Cu}$ & $\mathrm{H}$ & $\mathrm{Kv}$ & $\mathrm{Ku}$ & $\mathrm{Cu}$ & & $\mathrm{H}$ & $\mathrm{Kv}$ & $\mathrm{Ku}$ & $\mathrm{Cu}$ \\
\hline Porosity & 1 & 2 & 3 & 4 & 5 & 6 & 7 & 8 & 9 & 10 & 11 & 12 & 13 \\
\hline 0.1 & 4.44 & 4.55 & 4.66 & 17.42 & 5.73 & 5.95 & 6.16 & 22.83 & 3.08 & 4.84 & 4.99 & 5.14 & 16.24 \\
\hline 0.2 & 4.42 & 4.64 & 4.86 & 16.87 & 5.51 & 5.95 & 6.36 & 21.71 & 3.18 & 4.73 & 5.02 & 5.30 & 15.53 \\
\hline 0.3 & 4.44 & 4.79 & 5.11 & 16.38 & 5.36 & 6.01 & 6.61 & 20.66 & 3.30 & 4.67 & 5.11 & 5.51 & 14.88 \\
\hline 0.4 & 4.54 & 5.00 & 5.43 & 15.96 & 5.30 & 6.16 & 6.92 & 19.71 & 3.46 & 4.69 & 5.26 & 5.77 & 14.29 \\
\hline 0.5 & 4.74 & 5.33 & 5.86 & 15.66 & 5.37 & 6.43 & 7.34 & 18.88 & 3.67 & 4.80 & 5.51 & 6.11 & 13.81 \\
\hline 0.6 & 5.11 & 5.83 & 6.47 & 15.55 & 5.62 & 6.89 & 7.92 & 18.26 & 3.96 & 5.07 & 5.91 & 6.60 & 13.49 \\
\hline 0.7 & 5.79 & 6.68 & 7.42 & 15.82 & 6.20 & 7.68 & 8.83 & 18.00 & 4.41 & 5.60 & 6.59 & 7.36 & 13.47 \\
\hline 0.8 & 7.22 & 8.31 & 9.19 & 16.96 & 7.46 & 9.20 & 10.46 & 18.54 & 5.23 & 6.72 & 7.87 & 8.72 & 14.11 \\
\hline 0.9 & 11.34 & 12.80 & 13.88 & 21.33 & 11.03 & 13.18 & 14.62 & 21.88 & 7.31 & 9.79 & 11.22 & 12.18 & 17.03 \\
\hline
\end{tabular}

Experimental estimations result in values of the Kozeny constant approximately equal to five. It follows from Table 3 that the Happel's, Kvashnin's, and Kuwabara's models yield $k$ values that are equal about to five at porosities of $0.1-0.6$. In this case, the $k$ values are nearly identical for media composed of spherical particles or randomly oriented cylinders. The Cunningham/MehtaMorse's model yields $k$ values that differ substantially from those calculated from 5 .

Note, it was shown in [56] that the use of the Kuwabara boundary condition in contrast to other types of boundary conditions does not lead to contradictions when studying electrokinetic phenomena.

\section{Conclusions}

Cell models of the flow in porous media formed by partially permeable spherical particles or cylinders (fibers) are capable of describing filtration of liquid in porous membranes (layers) (porosity below 0.6) using the Happel's, Kvashnin's and Kuwabara's boundary conditions. Application of Cunningham/Mehta-Morse's boundary condition results in a substantial deviation from the previous models. However, additional well defined experiments are required to select 
between those models. We showed that cylindrical cell model works reasonably well for modeling the hydrodynamic permeability of membranes consisting of fibers and spherical cells adequately describe the behavior of membranes with globular structure.

This study theoretically verifies the previous experimental observations which conclude that the Kozeny constant is approximately equal to 5 for wide range of the membrane porosities.

Usage of the jump coefficient $\beta$ in the cell model proposed here gives possibility to take into account the influence of slipping between porous medium and clear liquid on the hydrodynamic permeability. The latter is probably important in the case of hydrophobic nanofiltration membranes.

\section{Acknowledgements}

Satya Deo is thankful to the DST New Delhi for sponsoring the work under the joint IndoRussian project (INT/RFBR/P-16). Ashish Tiwari acknowledge with thanks to UGC, New Delhi for the award of JRF No. 10-2 (5) /2005(i)-E.U.II for undertaking this research work. Research of Sergey Vasin and Anatoly Filippov was supported by the Russian Foundation for Basic Research (project 08-08-91306-IND_a). Victor Starov's research was supported by Engineering and Physical Sciences Research Council, UK and EU under Grant MULTIFLOW, FP7-ITN- 2008-214919. 


\section{List of main symbols}

Latin

$\tilde{a}$ Radius of inner solid cylindrical core

$\tilde{b}$ Radius of porous cylindrical particle

$\tilde{c}$ Radius of cylindrical cell

$\tilde{r}, \theta, \tilde{z}$ Cylindrical polar co-ordinates

$\tilde{F}$ Drag force

$\tilde{p}$ Fluid pressure at any point

$\tilde{v}_{\tilde{r}}, \tilde{v}_{\theta}$ Velocity components of fluid at any point

$\tilde{T}_{\tilde{r} \tilde{r}}$ Normal stress

$\tilde{T}_{\tilde{r} \theta}$ Tangential stress

$\tilde{U}$ Uniform velocity of fluid

$I_{n}(\xi)$ Modified Bessel functions of the first kind and of order $n$

$K_{n}(\xi)$ Modified Bessel functions of the second kind and of order $n$

$\tilde{k}$ Permeability

$k$ Kozeny's constant

$\tilde{\mathbf{v}}$ Fluid velocity at any point

$\widetilde{L}_{11}$ Hydrodynamic permeability of a membrane

$L_{11}$ Dimensionless hydrodynamic permeability of a membrane

$A_{1}, B_{1}, C_{1}, D_{1}, A_{2}, B_{2}, C_{2}, D_{2}$ Integration constants

Greek

$\alpha, \sigma$ Dimensionless parameters (see Eq (21)).

$\tilde{\mu}^{(1)}$ Viscosity of a clear fluid

$\tilde{\mu}^{(2)}$ Effective viscosity of porous medium

$\varepsilon$ Porosity

$\psi$ Stream function

$\gamma$ Particle volume fraction

$\lambda^{2}=\tilde{\mu}^{(2)} / \widetilde{\mu}^{(1)}$ Ratio of viscosities

$l=\tilde{a} / \tilde{b}$ and $m=\tilde{c} / \tilde{b}$ Dimensionless quantities (see Eq (21)) 
$\beta$ Jump coefficient

$\nabla^{2}$ Laplace operator 


\section{References}

1. J. Happel, H. Brenner, Low Reynolds Number Hydrodynamics, Martinus Nijoff, The Hague, Netherlands, 1983.

2. R. Varoqui, P. Dejardin, J. Chem. Phys. 66(10) (1977) 4395.

3. H.C. Brinkman, J. Appl. Sci. Res. A1 (1947) 27.

4. S. Veerapaneni, M.R. Wiesner, J. Colloid Interface Sci. 177 (1996) 45.

5. C. L. M. H. Navier. Memoire sur les lois du mouvement des Auides, Mt'moires de l'Academie Royale des Sciences de l'Institut de France V1 (1823) 389 - 440.

6. L. M. Hocking. A moving fluid interface on a rough surface. J: Fluid Mech. 76 (1976) 801 817.

7. H. Lamb. Hydrodynamics (Cambridge University Press, Cambridge 1932).

8. J. C. Maxwell. On stresses in rarefied gases arising from inequalities of temperature, Phil. Trans. R. Soc., 170 (1879) 231-256.

9. L. M. Hocking, $A$ moving fluid interface. Part 2. The removal of the force singularity by a slip flow, J. Fluid Mech. 79 (1977) 209--229.

10. C. Huh and L. E. Scriven. Hydrodynamic model of steady movement of a solid/liquid/fluid contact line, J. Colloid Interface Sci. 35 (1971) 85--101.

11. G. K. Batchelor. An Introduction to Fluid Dynamics (Cambridge University Press. Cambridge 2000).

12. O. I. Vinogradova. Drainage of a thin liquid film confined between hydrophobic surfaces. Langmuir 11 (1995) 2213-2220.

13. P. A. Thompson and M. O. Robbins. Shear flow near solids: epitaxial order and low boundary conditions, Phys. Rev. A 41 (1990) 6830-6837.

14. P. A. Thompson and S. M. Troian. A general boundary condition for liquid flow at solid surfaces, Nature 389 (1997) 360--362.

15. T. Qian. X. P. Wang and P. Sheng. Molecular scale contact line hydrodynamics of immiscible flows, Phys. Rev. E 68 (2003) 1-15.

16. T. Qian, X. P. Wang and P. Sheng. Power-law slip profile of the moving contact line in twophase immiscible flows, Phys, Rev. Lett. 93 (2004) 1-4.

17. T. Qian and X. P. Wang. Driven cavity flow: from molecular dynamics to continuum hydrodynamics, Mult. Mod. Sim. 3 (2005) 749 - 763.

18. M. T. Matthews, J. M. Hill. Flow around nanospheres and nanocylinders. Quart. J. Appl. Mech. Math. 59 (2006) 191 - 210.

19. S. Richardson. On the no-slip boundary condition. J. Fluid Mech. 59 (1973) 707-719. 
20. V. P. Sokhan, D. Nicholson and N. Quirke. Fluid flow in nanopores: accurate boundary conditions for carbon nanotubes, J. Chem. Phvs. 117 (2002) 8531 - 8539.

21. V. P. Sokhan, D. Nicholson and N. Quirke, Fluid flow in nanopores: an examination of hydro- dynamic boundary conditions, J. Chem. Phys. 115 (2001) $3878-3887$.

22. Solomentsev YuE, Starov VM, Colloid J, USSR Academy of Sciences, English Translation, (1990), 52, 785.

23. J.A. Ochoa-Tapia, S. Whitaker, Int. J. Heat Mass transfer 38 (1995) 2635.

24. J.A. Ochoa-Tapia and S. Whitaker, Int. J. Heat Mass transfer 38 (1995) 2647.

25. F. J. Valdes-Parada, B. Goyeau, J. A. Ochoa-Tapia. Jump momentum boundary condition at a fluid-porous dividing surface: derivation of the closure problem. Chem. Engng. Sci. 62 (2007) 4025-4039.

26. G.S. Beavers and D. D. Joseph. Boundary conditions at a naturally permeable wall, J.Fluid Mech. 30 (1967) 197-207.

27. A.N. Filippov, V.M. Starov, V.A. Lyalin. Gel Formation on a Surface of Ultrafiltration Membranes. Quasi Stationary Approach. Soviet J. Water Chemistry and Technology. 11(4) (1989) 291-296.

28. V.M. Starov, A.N. Filippov, V.A. Lyalin, I.V. Usanov. Gel-Layer Formation on a Surfaces of Ultrafiltration Membranes. Theory and Experiment. Soviet J. Water Chemistry and Technology. 12(4) (1990) 300-305.

29. E. K. Zholkovskiy, V. N. Shilov, J. H. Masliyah and M. P. Bondarenko. Hydrodynamic Cell Model: General Formulation and Comparative Analysis of Different Approaches. The Canadian Journal of Chemical Engineering. 85 (2007) 701-725.

30. J. Happel, A.I.Ch. E. 5(2) (1959) 174.

31. S. Kuwabara, J. Phys. Soc. Japan 14 (1959) 527.

32. A.G. Kvashnin, Fluid Dynamics 14 (1979) 598.

33. E. Cunningham, Proc. Roy. Soc. (London) A 83 (1910) 357.

34. G.D. Mehta, T.F. Morse, J. Chem. Phys. 63(5) (1975) 1878.

35. P.V. Perepelkin, V.M. Starov, A.N. Filippov. Permeability of Suspensions. Colloid J. of Russian Academy of Sciences, 54(2) (1992) 257-262 [139-145].

36. S.I. Vasin, V.M. Starov, A.N. Filippov. Motion of a Solid Spherical Particle Covered with a Porous Layer in a Liquid. Colloid J. of Russian Academy of Sciences, 58 (3) (1996) 282290 [298-306].

37. S.I.Vasin, V.M. Starov, A.N. Filippov. Hydrodynamic Permeability of a Membrane Regarded as a Set of Porous Particles (the Cell Model). Colloid J. of Russian Academy of Sciences, 58(3) (1996) 291-295 [307-311]. 
38. S. I. Vasin and A. N. Filippov. Hydrodynamic Permeability of the Membrane as a System of Rigid Particles Covered with Porous Layer (Cell Model). Colloid Journal, 66(3) (2004) 266-270 [305-309].

39. A.N. Filippov, S.I. Vasin, V.M. Starov, Colloids Surf. A Physicochem Eng. Asp. 282-283 (2006) 272.

40. S.I. Vasin, A.N. Filippov and V.M. Starov. Hydrodynamic permeability of membranes built up by particles covered by porous shells: Cell models. Adv. Colloid \& Interface Sci.139 (2008) 83.

41. G. Dassios, M. Hadjinicolaou, A.C. Payatakes, Q. Appl. Math. 52 (1994) 157.

42. G. Dassios, M. Hadjinicolaou, F.A. Coutelieris A.C. Payatakes, Int. J. Engng. Sci. 33(10) (1995) 1465.

43. S.I Vasin, A.N. Filippov. Permeability of Complex Porous Media. Colloid. J. Russ. Acad. Sci. 71 (2009) 31-45 (English Translation).

44. I.B. Stechkina, Fluid Dynamics 14(6) (1979) 912.

45. I. Pop, P. Cheng, Int. J. Engng. Sci. 30(2) (1992) 257.

46. S. Deo, Sadhana 29(4) (2004) 381.

47. D. Palaniappan, K. Archana, S.K. Khan, Z.A.M.M. 77(10) (1997) 791.

48. S. Datta, M. Shukla, Bull. Cal. Math. Soc. 95(1) (2003) 63.

49. S. Deo and P.K. Yadav, I.J.M.M.S. (2008), Article ID 651910 doi:10.1155/2008/651910 (published online).

50. S. I. Vasin and A. N. Filippov. Cell Models for Flows in Concentrated Media Composed of Rigid Impermeable Cylinders Covered with a Porous Layer. Colloid Journal. Vol. 71(2) (2009) 141-155 [149-163]

51. S. Deo, P. K. Yadav, A. Tiwari. Slow viscous flow through a membrane built up from porous cylindrical particles with an impermeable core. Applied Mathematical Modelling. 34 (2010) 1329-1343.

52. A.C. Srivastava, N. Srivastava, Z. Angew. Math. Phys. 56 (2005) 821.

53. A. Bhattacharya, G.P. Raja Shekhar, Z. Angew. Math. Phys. 56 (2005) 475.

54. Zhdanov VG, Starov VM. Advances in Colloid and Interface Science, 137 (2008) 2-19.

55. M. Abramowitz, I.A. Stegun, Handbook of Mathematical Functions, Dover Publications, New York, 1970.

56. E.K. Zholkovskij, J.H. Masliyah, V.N. Shilov, S. Bhattacharjee, Adv. Colloid Interface Sci. 134-135 (2007) 279. 